\title{
A miR-205-5p/GGCT/CD44 axis controls the progression of papillary thyroid cancer
}

\section{Han-ning Li ( $\nabla$ lihanninglhn@tjh.tjmu.edu.cn )}

Tongji Hospital of Tongji Medical College of Huazhong University of Science and Technology https://orcid.org/0000-0001-6281-6994

\section{Hui-min Zhang}

Wuhan University of Science and Technology

\section{Xing-rui Li}

Tongji Hospital of Tongji Medical College of Huazhong University of Science and Technology Jun Wang

Wuhan University of Science and Technology

\section{Tao Xu}

Tongji Hospital of Tongji Medical College of Huazhong University of Science and Technology

\section{Shu-yu Li}

Tongji Hospital of Tongji Medical College of Huazhong University of Science and Technology

\section{Meng-lu Dong}

Tongji Hospital of Tongji Medical College of Huazhong University of Science and Technology

\section{Ge Wang}

Tongji Hospital of Tongji Medical College of Huazhong University of Science and Technology

\section{Xiao-qing Cui}

Tongji Hospital of Tongji Medical College of Huazhong University of Science and Technology

\section{Xue Yang}

Tongji Hospital of Tongji Medical College of Huazhong University of Science and Technology

\section{Yong-lin Wu}

Tongji Hospital of Tongji Medical College of Huazhong University of Science and Technology

\section{Xing-hua Liao}

Wuhan University of Science and Technology

\section{Ya-ying Du}

Tongji Hospital of Tongji Medical College of Huazhong University of Science and Technology

\section{Research}

Keywords: GGCT, miR-205-5p, PTC, CD44

Posted Date: October 18th, 2021 
DOl: https://doi.org/10.21203/rs.3.rs-965023/v1

License: (c) (1) This work is licensed under a Creative Commons Attribution 4.0 International License. Read Full License 


\section{Abstract \\ Background}

Papillary thyroid cancer (PTC) is the most common endocrine malignancy, despite marked achieves in recent decades, the mechanisms underlying the pathogenesis and progression for PTC are incomplete. Accumulating evidence shows that $\gamma$-glutamylcyclotransferase (GGCT), an enzyme participated in glutathione homeostasis that is elevated in multiple types of tumors, represents an attractive therapeutic target.

\section{Methods}

Bioinformatics, immunohistochemistry (IHC), qRT-PCR and western blot (WB) assays were used to determine the elevation of GGCT in PTC. The biological functions of GGCT were examined using CCK8, wound healing and transwell assays. Subcutaneous xenograft and tail vein pulmonary metastatic mouse models were constructed to determine the effect of GGCT on tumorigenicity and metastasis in vivo. The effect of miR-205-5p on GGCT and the relationship between these two molecules were examined by dual luciferase reporter assay, RNA-RNA pull down assay as well as the rescue experiments both in vitro and in vivo. The interaction between GGCT and CD44 was assessed by co-immunoprecipitation (Co-IP) and IHC assays.

\section{Results}

Our results showed that GGCT expression is upregulated in PTC, correlates with more aggressive clinicopathological characteristics and worse prognosis. GGCT knockdown inhibited the cell proliferation, migratory and invasion ability of PTC cells and reduced the expression of mesenchymal markers ( $\mathrm{N}$ cadherin, CD44, MMP-2 and MMP9) while increased epithelial marker (E-cadherin) in PTC cells. We confirmed binding of miR-205-5p on the 3'-UTR regions of GGCT and delivery of miR-205-5p reversed the pro-malignant capacity of GGCT both in vitro and in vivo. Lastly, we found GGCT interacted with and stabilized CD44 in PTC cells.

\section{Conclusions}

Our findings illustrate a novel signaling pathway, miR-205-5p/GGCT/CD44, that involves in the carcinogenesis and progression of PTC. Development of miR-205-mimics or GGCT inhibitors as potential therapeutics for PTC may have remarkable applications.

\section{Background}


Papillary thyroid cancer (PTC) represents the most commonly occurring type of differentiated thyroid carcinoma, accounting for approximately $80-90 \%$ of all thyroid cancer patients, with rapidly rising incidence during the last several decades worldwide[1, 2]. Despite common perceptions that PTC possesses indolent behavior and carries relatively low disease-specific mortality, locoregional relapse and distant metastases occur in up to $20 \%$ and $10 \%$ cases at ten years respectively, which causes enormous impacts on the survival of these patients[3, 4]. Based on molecular profiles, PTC can be roughly grouped into two distinct subclasses: BRAFV600E-like PTCs (harboring BRAFV600E mutations and showing insistent activation of MAPK signaling pathway) and RAS-like PTCs (characterizing by K/H/N-RAS mutations and activating in both the MAPK and PI3K/AKT pathways) [5]. Nevertheless, with the deepening of the research, a series of novel onco-driver genes in PTC have been identified[6-8], which prompts the existence of a much more complex regulatory network in the occurrence and progression of PTC and calls for the importance of revealing the pathogenesis of this condition.

An enzyme involved in glutathione metabolism cycle, $y$-glutamylcyclotransferase (GGCT) catalyze the reaction that induces 5-oxoproline and free amino acids generation from the $y$-glutamyl peptide[9]. Intriguingly, growing evidence substantiates a vital role of GGCT in oncogenesis. GGCT was identified to be highly expressed in several carcinomas, including bladder cancer[10], breast cancer[11], ovarian cancer[12] and gastric cancer[13]. RNA interference (RNAi)-mediated knockdown of GGCT weakens proliferation of various tumor cell lines without disturbing growth of normal cells[14]. Moreover, GGCT is involved in the shift of oxidative phosphorylation to aerobic glycolysis by regulating the expression of HIF-1a in multiple tumors, which makes tumor cells adapt better to the hypoxia environmental stresses[15]. Additionally, GGCT is required for oncogenic K-RAS-driving lung tumor formation and the scavenge of exceeding reactive oxygen species (ROS) in mice[16]. However, studies on the functions of GGCT in PTC have not been reported.

MicroRNAs are a group of endogenous small RNAs that control gene expression in various biological processes such as cell differentiation, proliferation and apoptosis[17]. MicroRNAs can prevent the translation of target mRNA or promote its degradation by binding to the 3 untranslated regions ( 3 '-UTR) of mRNA in a complementary manner[18]. In human cancers, both carcinogenic or tumor suppressor roles of microRNA have been proposed, based on the distinct functions of substrate genes[19, 20]. Of particular concern is that the initiation and progression of PTC are often accompanied by the alteration of microRNA profiles[21]. MiR-205-5p, a novel microRNA, has been reported to reduce angiogenesis and suppress cell proliferation via post-transcriptional manipulation of VEGFA in PTC[22]. Another study found a DLEU2/miR-205-5p/TNFAIP8 signaling axis involving in the regulation of the proliferation, migration and aerobic glycolysis processes in PTC cells[23]. Interestingly, later clinical simple based research suggests miR-205-5p combined with TSHR mRNA could be used to distinguish benign thyroid nodules from malignant ones[24]. These highlights the potential of miR-205-5p to serve as a candidate molecular for both diagnosis and treatment of PTC.

In this study, we comprehensively evaluated GGCT expression, its relationship with clinicopathological characteristics and prognosis, and its effect on PTC cell's biological behaviors through clinical samples 
and loss-of-function assays. Moreover, the role of miR-205-5p as an upstream negative regulator of GGCT has been confirmed through dual-luciferase reporter assay, RNA pull-down assay as well as genetic rescue experiments. Lastly, we found GGCT can bind to CD44 and maintain protein stability of CD44, the latter of which has been shown to increase the malignant potential of tumor cells[25]. Our study shed light on a novel signaling pathway, miR-205-5p/GGCT/CD44, which play critical roles in cell proliferation and metastasis of PTC.

\section{Materials And Methods}

\section{Antibody information}

Antibodies against GGCT (ab198503), E-cadherin (ab227639), N-cadherin (ab76011), CD44 (ab189524), MMP2 (ab92536), MMP9 (ab76003) were purchased from Abcam Biotechnology Co, Ltd. (USA). Antibodies against Flag-tag (14793S), His-tag (12698S), GAPDH (5174S), $\beta$-actin (4970S) were bought from Cell Signaling Technology, Inc. (USA).

\section{Cell culture and patient specimens}

Human normal thyroid cell line Nthy-ori 3-1; PTC cell lines TPC-1, BCPAP, K1; human embryonic kidney 293T (HEK293T) cell line were purchased from Shanghai Cell Bank of Chinese Academy of Sciences (China). Nthy-ori 3-1, TPC-1, and BCPAP were cultured in RPMI 1640 medium (Gibco, USA), while K1 and HEK293T were cultured in RPMI DMEM medium (Gibco, USA). All media contained $10 \%$ fetal bovine serum (FBS) (Gibco, USA) and 1\% penicillin-streptomycin (meilunbio, China) and all cells were kept in a $37^{\circ} \mathrm{C}, 5 \%$ carbon dioxide cell incubator.

The samples of carcinomas $(n=176)$ and the adjacent normal thyroid tissues $(n=82)$ were obtained from surgical specimens from patients with PTC who were hospitalized at Tongji hospital (Wuhan, China) between 2017.1 and 2020.10. The whole blood samples were collected prior to surgery and $24 \mathrm{~h}$ after surgery from each patient and were used for serum extraction by centrifugation. Fresh specimens were formalin-fixed and paraffin-embedded or directly frozen into liquid nitrogen for further analysis. The patients enrolled received no prior drug or radioiodine ablation therapy before the surgical intervention. An informed consent was obtained from each participant and the study protocol was approved by the Ethics Committee of Tongji Hospital, Tongji Medical College, Huazhong University of Science and Technology, and followed the ethical guidelines of the Declaration of Helsinki.

\section{Quantitative real-time polymerase chain reaction (qRT-PCR)}

Total mRNA and MicroRNA of cells or tissues were extracted with Ultrapure RNA Kit (CWBIO, China) and miRNA Purification Kit (CWBIO, China) respectively. HiScript ${ }^{\circledR}$ II 1st Strand cDNA Synthesis Kit (+gDNA wiper) (Vzayme, China) or miRNA 1st Strand cDNA Synthesis Kit (by stem-loop) (Vzayme, China) was used for reverse transcription of mRNA or MicroRNA, respectively. Subsequently, qPCR SYBR Green (YEASEN, China) was used for fluorescence quantitative analysis in the Bio-Rad CFX96 Touch real-time fluorescent quantitative PCR system (USA). U6 and GAPDH are used as internal reference genes for 
miRNA and mRNA respectively. The qPCR primers involved in this study are provided in Table.S1 of Supplementary Materials. The $2^{-\Delta \Delta C t}$ method was utilized to quantify gene expression.

\section{Western blot analysis}

The RIPA Lysis Solution (Beyotime, China) was used to extract the total protein and the BCA kit (Beyotime, China) was used to quantify the protein concentration. $30 \mu \mathrm{g}$ of total protein was separated in $10 \%$ polyacrylamide gel, and then the target protein was transferred to polyvinylidene fluoride (PVDF) membrane, and blocked with 5\% skim milk dissolving in tris-buffered saline with Tween-20 (TBST) at room temperature for $1 \mathrm{~h}$. The primary antibody was incubated overnight at $4^{\circ} \mathrm{C}$ and then washed with TBST. The secondary antibody incubation was then performed at room temperature for $1 \mathrm{~h}$. The blots were exposed digitally using an ChemiDoc Imaging System (BioRad, USA) and target bands were quantified using Image Lab Software (version 6.0, BioRad, USA).

\section{Human serum and enzyme-linked immunosorbent assays (ELISA)}

Human serum was separated and collected from whole blood following centrifugation at $1500 \times \mathrm{g}$ for 10 min. The level of secreted GGCT was quantified by a human ELISA kit (RX100080H; Ruixin Biotechnology Co, Ltd., China) in strict accordance with the manufacturer's instructions.

\section{Plasmid construction and lentivirus}

The GGCT 3- 'UTR sequence was amplified from the human genome, and the fragment was connected to pmirGLO (Addgene, USA) to obtain the wild-type luciferase reporter plasmid (WT-pmirGLO-GGCT). Using WT-pmirGLO-GGCT as a template, the binding site of miR-205-5p and GGCT 3- 'UTR was mutated to obtain a mutant luciferase reporter plasmid (MUT-pmirGLO-GGCT). The coding sequence of CD44 or GGCT was amplified using human CDNA as a template, and a $6 \times$ His-tag was added to the C-terminal of CD44, while a $3 \times$ Flag-tag was inserted to the N-terminal of GGCT. The obtained fragments were connected to pcDNA3.1 (Addgene, USA). For lentivirus-Mediated gene silencing, we designed three distinct short hairpin RNAs (shRNAs) oligonucleotides for the target sequence of GGCT (shRNA\#1: GATTATTTGCATGGGTGCAAA, shRNA\#2: GCAATAGAACCAAATGACTAT, shRNA\#3:

GCTGGAGTATCAAGAGAAGTT). Non-targeting control of shRNA (NC) was also synthesized. The above oligonucleotides were inserted into the pLKO.1 vector. Lentivirus vectors expressing pre-miR-205 and miR205-NC were obtained from GeneChem (Shanghai, China). For stable GGCT overexpression, GGCT gene was amplified and inserted into PLVX-EF1a-IRES-puro to generate recombinant pLVX-GGCT for lentivirus production.

\section{Cell counting kit-8 (CCK8) assay}

Cell proliferation was monitored with a CCK8 kit (Dojindo, Japan) following the producer's instructions. PTC cells $\left(1 \times 10^{3}\right.$ cells per well) were seeded in a 96-well plate and cultivated for the indicated times. Then, $10 \mu \mathrm{L}$ CCK8 solution was added to each well with incubation $2 \mathrm{~h}$ before analysis. The absorbance 
$(450 \mathrm{~nm})$ of each well was determined using a multifunctional microplate reader (Thermo Fisher Scientific, USA).

\section{Wound-healing assay}

$1 \times 10^{6}$ thyroid cancer cells were seeded into a 6 -well plate. When the cell confluence reaches about $90 \%$, scratch the cells with a $200 \mu \mathrm{L}$ sterile pipette tip, and replace the medium to reduced serum medium containing $0.2 \%$ FBS to maximally maintain cell survival but limit cell proliferation. Areas of the wound gap were assessed and photographed at $0 \mathrm{~h}, 24 \mathrm{~h}$ or $48 \mathrm{~h}$ after wound generation with an inverted microscope (Olympus, Japan), and the gap areas were quantified by using Image $\mathrm{J}$ software $(\mathrm{NIH}, \mathrm{USA})$. The experimental results were obtained after three independent repetitions.

\section{Transwell invasion assay}

The bottom of the transwell chamber $(8 \mu \mathrm{m}$, CORNING, USA) used in the experiment was covered with Matrigel ( $1: 50$ dilution, $B D, U S A)$, and the cells were serum-deprived for $12 \mathrm{~h}$, and then $3 \times 10^{4}$ cells were inoculated into the upper chamber of the transwell with $5 \%$ FBS in $150 \mu \mathrm{L}$ medium. Add $600 \mathrm{ul}$ medium containing $15 \%$ FBS to the lower chamber. After incubation for $24 \mathrm{~h}$, non-invading cells were wiped off with a cotton swab and invading cells were stained using $0.1 \%$ crystal violet, $4 \%$ paraformaldehyde in PBS for 20 min. An inverted microscope (Olympus, Japan) was used to visualize the invaded cells, and five different fields with a 10x objective were counted from each invasion chamber independently. The experimental results obtained were repeated three times independently.

\section{Dual-luciferase reporter assay}

WT-pmirGLO-GGCT or MUT-pmirGLO-GGCT and miR-205 mimics were co-transfected into HEK293T cells, and the cells were harvested $24 \mathrm{~h}$. According to the instructions of the Luciferase Reporter Gene Detection Kit (Meilun, China), add cell lysis buffer to the cells and lyse for 10 minutes on ice. After centrifugation at $12000 \mathrm{~g}$ for $5 \mathrm{~min}, 20 \mu \mathrm{L}$ of supernatant was added to $100 \mu \mathrm{L}$ Firefly Luciferase Reaction Buffer and Renilla Luciferase in sequence; Firefly Luciferase and Renilla Luciferase reporter gene activities were read in the microplate reader (Bio-Rad, USA) respectively. The experimental data was obtained after three independent repetitions.

\section{Biotin RNA-RNA pull down assay}

K1 cell lysates were prepared and incubated with biotinylated double-stranded RNA of wild type (WT) miR-205-5p (Bio-miR-205-5p-WT) or mutant miR-205-5p (Bio-miR-205-5p-WUT) or control random RNA (Biotin-NC) for $2 \mathrm{~h}$ at $4{ }^{\circ} \mathrm{C}$ to form RNA-RNA complexes. The coupled complexes were captured by magnetic beads conjugated with streptavidin (Dynabeads ${ }^{\mathrm{TM}}$ MyOne ${ }^{\mathrm{TM}}$ Streptavidin C1, Thermo Fisher Scientific, USA). Finally, the complexes of miRNA/mRNA were eluted and the mRNA expression of GGCT was assessed by qRT-PCR.

\section{Endogenous and exogenous co-immunoprecipitation (Co- IP) assays}


For endogenous Co-IP assays, K1 cells were lysed in 1mL Cell lysis buffer for IP (RM00022, ABclonal, China) at $4^{\circ} \mathrm{C}$ for 20 minutes. The input group contains 10 ul protein supernatant mixed with an equal volume of $2 X$ loading buffer. The remaining protein supernatant was divided into two equal parts and added Mouse Control IgG (AC001, ABclonal, China) or Anti-GGCT (ab198503, Abcam) respectively, and inverted the mixer at $4^{\circ} \mathrm{C}$ for 2 hours. Wash rProtein A/G Plus MaqPoly Beads (RM09008, ABclonal, China) twice with Cell lysis buffer for IP and block with 3\% BSA at 4 degrees Celsius for 1 hour. The antibody-antigen binding complex is mixed with the ready-for-use magnetic beads, and reacted at $4^{\circ} \mathrm{C}$ for 2 hours. Put the above-mentioned magnetic bead-antibody-antigen complex on a magnetic separator for separation and wash twice. Add 30 $\mu$ I X SDS-PAGE Loading Buffer to it and mix, and heat at $95^{\circ} \mathrm{C}$ for $15 \mathrm{~min}$. Place it on a magnetic separator for magnetic separation, and collect the supernatant for SDSPAGE detection.

For exogenous detection, the HEK293T cell group was divided into three groups that overexpress FlagGGCT or His-CD44 or co-transfected with Flag-GGCT and His-CD44. After the cells are lysed in the cell lysis buffer, a portion of the supernatant is used for the input group, and the remaining protein supernatant is respectively incubated with Anti-Flag (14793S, CST) or Anti-His (12698S, CST) for antigenantibody incubation. After the magnetic beads-antibody-antigen complex incubation, SDS-PAGE detection was performed.

\section{Animal experiment}

Four-week-old nude mice were purchased from Model Animal Research Center of Nanjing University (Nanjing, China). All nude mice are kept in specified-pathogen-free (SPF) environment, and all experiments in animals were approved by the Animal Ethics Committee of Wuhan University of Science and Technology.

For subcutaneous tumor xenograft model, stable knockdown (shControl and shGGCT) and overexpression (Lenti-PLVX, Lenti-GGCT, Lenti-miR-205, Lenti-GGCT+miR-205) PTC cell lines were generated via the lentiviral system as described above. Total of $5 \times 106$ cells of PTC cells resuspended in $100 \mu \mathrm{l}$ PBS were injected into the right axillary fossa of nude mice. The length and width of the subcutaneous tumor was measured with a vernier caliper every 7 days after inoculation. The formula for detecting the tumor volume is: volume $\left(\mathrm{mm}^{3}\right)=0.5 \times$ width ${ }^{2} \times$ length. 28 days after the inoculation, the subcutaneous tumors were harvested and the tumor quality was counted. The subcutaneous tumor tissues were subjected to HE staining, immunohistochemistry, and western blot analysis.

For tail vein lung metastatic model, $\mathrm{K} 1$ cells were stably transfected with firefly-luciferase (fLuc) gene by lentivirus. Mice were then injected with $1 \times 106$ fLuc-labeled K1 cells via the tail vein. Growth of pulmonary K1-fLuc metastases was monitored by bioluminescence imaging (BLI) every 7 days. Mice were anesthetized with 2-3\% isoflurane and intraperitoneally injected with $150 \mathrm{mg} / \mathrm{kg}$ D-luciferin potassium salt (Yeason, Shanghai, China). Tumors exhibiting fLuc expression were imaged 10 min postinjection with a Xenogen IVIS Lumina system (Caliper Life Sciences). On 21th day after the initial inoculation, the mice were euthanized, the visceral organs were gently removed and used for ex vivo BLI. 


\section{Hematoxylin-eosin (HE) staining and immunohistochemistry (IHC)}

For human and mouse histological examination, tissues were fixed in 10\% neutral buffered formalin for 24h. All simples were paraffin-embedded and sliced to $4 \mu \mathrm{m}$. HE staining was performed by Servicebio Biotechnology Co. Ltd. (Wuhan, China) according to standard protocols. For IHC, the sections were deparaffinized, rehydrated and followed by antigen repaired with citrate antigen repair buffer $(\mathrm{pH} 6.0)$, and then, subjected to endogenous peroxidase inactivation with $3 \%$ hydrogen peroxide for $15 \mathrm{~min}$ at room temperature. After washing, unspecific antigen binding was blocked with $10 \%$ normal goat serum for $30 \mathrm{~min}$ at $37^{\circ} \mathrm{C}$ and probed with indicated primary antibodies overnight at $4^{\circ} \mathrm{C}$ before HRP-conjugated secondary antibodies incubation for $45 \mathrm{~min}$ at room temperature. Chromogen detection was conducted with the 3,3'-diaminobenzidine (DAB) chromogen kit (Servicebio, Wuhan, China). Nuclei were counterstained with hematoxylin. The IHC results were evaluated using following criteria: the intensity score was judged by a scale of $0-3$ points: 0 for no staining, 1 for weak staining, 2 for moderately positive staining, 3 for strongly positive staining; the percentage of positive areas was judged by a scale of $0-4$ points: $0 \%(0),<10 \%(1), 10-30 \%(2), 31-70 \%(3), 71-100 \%$ (4). The final score of IHC was determined through multiplying the intensity score by the percentage score to obtain a maximum of 12 . In addition, high-GGCT expression was defined as IHC score $\geq 6$, whereas low-GGCT expression was defined as IHC score $<6$.

\section{Data analysis}

All statistical analyses were performed using R software (R Core Team, Version 4.0.2) or GraphPad Prism (version 7.0). For the continuous variables, Student's t-test was utilized to analyze the statistical significance of the differences between groups and the data were represented by the mean \pm S.D. Fisher exact tests and Chi-square tests were used to determine the correlation between GGCT and the clinicopathological parameters. Survival was analyzed by Kaplan-Meier methods and presented as Kaplan-Meier curves. Linear regression analysis was used for gene correlation analysis. Significance between groups was represented by $\left.{ }^{\star} p \llbracket 0.05,{ }^{\star \star} p \llbracket 0.01,{ }^{\star \star \star} p \llbracket 0.001\right)$.

\section{Results}

\section{GGCT is overexpressed in PTC tissues and cell lines.}

To explore the expression pattern of GGCT in PTC, we first detected its expression in 27 pairs of PTC tissues and paracancerous tissues by RT-qPCR. As illustrated in Figure 1A, PTC tissues exhibited prominently elevated GGCT mRNA expression as compared to the corresponding paracancerous tissues. These data were also confirmed in the TCGA database, in where GGCT was significantly higher in tumor tissues than in normal ones for both unpaired (Supplementary Figure 1A) and paired (Supplementary Figure 1B) simple comparisons. Additionally, we further assessed GGCT protein level in 6 pairs of PTC and non-cancerous tissues using western blot analysis which showed most PTC tissues possessed 
greater GGCT expression than the normal controls (Figure 1B and 1C). Similar findings were also obtained with paraffin-embedded sections including 178 PTC and 82 normal thyroid tissues by IHC. As shown in Figure 1D and 1E, higher expression of GGCT was observed in the PTC tissues compared with the normal counterparts. $(P<0.05)$. Besides, we measured a range of thyroid-derived cell lines for GGCT expression, and as expected, high levels of GGCT expression were visible in all three PTC cell lines but not in the normal thyroid follicular epithelial cell line (Nthy-ori-3-1) (Figure 1F and 1G). As GGCT has been estimated to have an elimination half-life in serum of up to 30 hours (https://web.expasy.org/cgibin/protparam/), we compared the blood content of GGCT preoperative and 24 hours postoperative from the same patient $(n=26)$, and the results clearly indicated the serum GGCT level was noticeably reduced after surgical intervention (Figure $1 \mathrm{H}$ ). Together these results strongly suggest that GGCT is upregulated in the PTC tissues and cell lines.

\section{High-level of GGCT correlates with unfavorable clinicopathological characteristics and worse outcomes.}

We focused on whether GGCT overexpression was responsible for worse PTC prognosis. Based on the IHC staining score, the patients were classified into the low-GGCT $(n=67)$ and high-GGCT expression group $(n=111)$. The Fishers exact or chi-square test showed that GGCT expression was strongly associated with tumor histological type $(p<0.001)$, extrathyroidal extension $(p=0.035)$, primary tumor classification ( $p=0.004)$, and TNM stage $(p=0.002)$ (Table 1). In addition, Kaplan-Meier survival curve revealed a shorter disease-free survival (DFS) time in high-GGCT expression group than in low-GGCT expression group (Supplementary Figure 2). These results infer that GGCT is closely correlated with tumor malignant properties and decreases DFS in PTC patients. 
Table 1

Relationship between GGCT expression and clinicopathological characteristics in 178 PTC tissues.

\begin{tabular}{|c|c|c|c|c|}
\hline \multirow[t]{2}{*}{ Clinicopathologic parameters } & \multirow[t]{2}{*}{$\mathbf{n}$} & \multicolumn{2}{|c|}{ GGCT expression } & \multirow[t]{2}{*}{$p$} \\
\hline & & low & high & \\
\hline All cases & 178 & 67 & 111 & \\
\hline Age & & & & 0.848 \\
\hline$\leq 55$ & 60 & 22 & 38 & \\
\hline$>55$ & 118 & 45 & 73 & \\
\hline Gender & & & & 0.159 \\
\hline male & 69 & 20 & 49 & \\
\hline female & 119 & 47 & 72 & \\
\hline Histological type & & & & $<0.001$ \\
\hline classical & 106 & 39 & 67 & \\
\hline follicular & 28 & 19 & 9 & \\
\hline tall cell & 44 & 9 & 35 & \\
\hline Multifocality & & & & 0.218 \\
\hline unifocal & 129 & 45 & 84 & \\
\hline multifocal & 49 & 22 & 27 & \\
\hline Extrathyroidal extension & & & & 0.035 \\
\hline no & 133 & 56 & 77 & \\
\hline yes & 45 & 11 & 34 & \\
\hline T classification & & & & 0.004 \\
\hline T1-T2 & 117 & 53 & 64 & \\
\hline T3-T4 & 61 & 14 & 47 & \\
\hline Lymph node metastasis & & & & 0.395 \\
\hline no & 87 & 30 & 57 & \\
\hline yes & 91 & 37 & 54 & \\
\hline TNM stage & & & & 0.002 \\
\hline $1+||$ & 128 & 57 & 71 & \\
\hline
\end{tabular}




\begin{tabular}{|llccl|}
\hline Clinicopathologic parameters & $\mathbf{n}$ & GGCT expression & $\boldsymbol{p}$ \\
\hline III+IV & 50 & 10 & 40 & \\
Braf-V600E mutation & & & & 0.975 \\
\hline no & 72 & 27 & 45 & \\
yes & 106 & 40 & 66 & \\
\hline
\end{tabular}

Knockdown of GGCT alleviates the proliferation, migration and invasion of PTC cells in vitro and in vivo.

Given the findings that upregulation of GGCT was associated with poorly clinicopathological characteristics and worse outcomes in PTC patients, we postulated that GGCT might act as a tumor facilitator in PTC. To assess the effect of GGCT on the biological behaviors of PTC cells, we performed the RNA interference experiment with three distinct shRNA duplexes. Among them, shRNA-3 exhibited the best knockdown efficiency, as indicated by WB analysis, and thus selected for the subsequent investigations (Figure 2A). Firstly, we assessed the impact of GGCT on cell growth through the CCK-8 assay. As shown in Figure 2B and 2C, GGCT knockdown remarkably inhibits the proliferation of K1 and BCPAP in a time-dependent manner. We next evaluated the effect of GGCT on cell migration and invasion via wound healing and Matrigel Transwell assays. The wound healing assay demonstrated that silencing of GGCT significantly retarded the closure of the wound gap after 24 hours and 48 hours (Figure 2D and $2 \mathrm{E})$. Also, shRNA-mediated abrogation of GGCT impeded the matrix penetration capability of K1 and BCPAP cell lines (Figure 2F and $2 \mathrm{G}$ ).

Since epithelial-mesenchymal transition (EMT) has previously been proved to be critical for the survival and invasiveness acquisition of cancer epithelial[26]. We questioned whether interference with GGCT expression inhibits cell proliferation, migration and invasion by reversing the EMT phenotype. Indeed, after stably downregulation of GGCT in PTC cells, K1 and BCPAP cells underwent several morphologic alterations from mesenchymal spindle-like phenotype to epithelial polarized phenotype in contrast to the control cells (data not shown). Subsequently, the EMT-related proteins were examined by WB analysis, and the results showed a downregulation of mesenchymal markers (N-cadherin, CD44, MMP-2, MMP-9) and a concomitant upregulation of epithelial marker (E-cadherin) in sh-GGCT cells, heralding the EMTpromoting role of GGCT in PTC (Figure $2 \mathrm{H}$ ).

To determine whether GGCT is required for in vivo tumor growth and metastasis, the nude mouse subcutaneous xenotransplant tumor model and the tail vein-lung metastasis model was performed. As pointed out in Figure 3A-C, GGCT-knockdown K1 cells exhibited an evidently decreased tumor volume and tumor weight 3 weeks post-modeling compared with the control group. Similar results were yielded by the xenografts constructed using BCPAP cell lines which were stably infected with sh-GGCT or sh-NC lentivirus (Figure 3D-F). Moreover, the efficiencies of GGCT knockdown in vivo and the EMT-associated markers were assessed by WB analyses, which proved the silencing of GGCT contributed to the reduction of tumor growth and reversion of EMT process (Supplementary Figure 3). As for the experimental 
pulmonary metastasis assay, sh-GGCT-K1 or sh-NC-K1 cells expressing firefly luciferase were administered via tail vein injection. The successful aggregation of tumor cells into the lung in both shGGCT and sh-NC groups was confirmed at day 0 by bioluminescence imaging (Figure 3G). With extended observation time, the bioluminescence signals in the sh-NC-K1 group rapidly increased and all mice in this group were forced to be sacrificed due to the tumor-associated cachexia up to 3 weeks post injection (Figure 3G-H). Surprisingly, none of the positive signals were captured in the sh-GGCT-K1 group throughout the observation period, demonstrating an entire abrogation of the formation of lung metastatic foci by GGCT repression (Figure 3G-H). These were also corroborated by an ex vivo imaging of the visceral organs in combination with HE staining of lung tissues (Figure $3 \mathrm{I}-\mathrm{J}$ ). All above shed light on that abolishment of GGCT weakened the malignant potential of PTC cells in vitro and in vivo by reversing the EMT process.

\section{MiR-205-5p can directly bound to the 3'UTR of GGCT and regulate GGCT expression.}

Evidences support the crucial role of miRNAs in various cancers by inhibiting their target genes[27]. Interestingly, mir-205-5p, a potential tumor suppressor in multiple malignancies, was predicted to contain the binding site of the 3UTR region of GGCT, as obtained by bioinformatics (Figure 4A). Further exploration showed that decreased expression of GGCT was observed in K1 cells transfected with mir205-5p mimics, while increased expression in mir-205-5p inhibitor groups (Figure 4B). To probe whether GGCT was the direct target of miR-205-5p, GGCT-3UTR with a wild type (WT) or mutated (MUT) miR-205$5 p$ binding motif was constructed and subcloned into the dual-luciferase vector (Figure 4C). It was found that miR-205-5p reduced the luciferase activity of WT 3UTR-reporter, but not the MUT 3'UTR-reporter (Figure 4D). Moreover, biotin RNA-RNA pull-down assay revealed that GGCT mRNA were significantly enriched by using Bio-miR-205-5p-WT than Bio-miR-205-5p-MUT and Bio-NC (Figure 4E-F), indicating the interactions between miR-205-5p and GGCT.

\section{MiR-205-5p reverses the pro-malignant phenotypes induced by GGCT dysregulation.}

To corroborate that mir-205-5p reverses the GGCT-induced promotion of cell proliferation and invasion, pre-miR-205 was introduced into K1-GGCT cells by lentiviral overexpression. Overexpressing of miR-205 resulted in an attenuated proliferation ability of K1-GGCT cells (Figure 5A). Also, upregulation of miR-205 in K1-GGCT cells leads to the inhibition of migratory (Figure 5B-C) and invasive (Figure 5D-E) capacities, accompanying with increased expression of the epithelial markers and decreased mesenchymal markers (Figure 5F). To further confirm if miR-205-5p eventually restores GGCT-mediated tumorigenicity and metastasis in vivo, K1-GGCT cells with or without miR-205 overexpression were administrated into immune-deficient nude mice. Introduction of miR-205 not only repressed tumor growth (Figure 5G-H), but diminished the metastatic lesions in the metastatic model (Figure 51-J). Altogether, these findings revealed that miR-205-5p suppressed the tumorigenicity and metastasis of PTC by targeting GGCT.

GGCT interacts with CD44 and inhibits the degradation of CD44. 
Considerable evidence indicates that the adhesion molecule CD44 plays a vital role in mediating chemoresistance, cell proliferation and EMT[25]. As data preceding indicates that CD44 expression was positively influenced by GGCT, we were therefore intrigued by which mechanism this phenomenon happens. As depicted in Figure 6A, overexpression of GGCT significantly upregulated the CD44 protein, but leave no effect on the mRNA level of CD44. Subsequently, co-immunoprecipitation assays were conducted in K1 cell extracts. We co-expressed CD44-his with vector or GGCT-flag in HEK293T cells. We saw a co-precipitated CD44-his when GGCT-flag was pulled down (Figure 6B). Also, the interaction was further substantiated by reciprocal co-immunoprecipitations (Figure 6C). Consistently, by immunoprecipitating GGCT with anti-GGCT, CD44 protein was successfully precipitated, confirming the existence of an endogenous GGCT-CD44 complex in K1 cells (Figure 6D). Subsequently, a cycloheximide (CHX) chase experiment was conducted to explore the function of GGCT on CD44 stability by blocking de novo protein biosynthesis. Exposure to $\mathrm{CHX}$ results in obviously degradation of CD44 protein in HEK293T cells transfected with GGCT-vector, but not in cells transfected with GGCT (Figure 6E). Furthermore, to evaluate the relationship of GGCT and CD44 expression, we carried out an immunohistochemical analysis of human PTC simples, and observed that CD 44 is positively correlated with GGCT protein levels (Figure 6F-G). Finally, an analysis of public databases demonstrated that high expression of CD44 is correlated with worse DFS of patients with PTC (Figure 6H). Collectively, these data signified that GGCT positively regulates CD44 expression by inhibiting its protein degradation.

\section{Discussion}

In the present study, we identified a novel miR-205-5p/GGCT/CD44 signaling pathway, which plays a momentous functional role in PTC pathogenesis. High level of GGCT was tightly correlated with that of more aggressive clinical characteristics and worse outcomes. GGCT depletion by RNA-interference mediated proliferation, migration, invasion and EMT suppressing effect of PTC cells in vitro, and reduced tumor growth and metastasis to lung in murine xenograft and tail vein metastatic models, suggesting that GGCT might function as a tumor facilitator. The mechanism of action linking GGCT and miR-205-5p lies in the directly binding to the 3'UTR of GGCT, which subsequently leads to transcriptional repression of GGCT expression and counteracts the pro-oncogenic effect of this protein. In addition, we found that $\mathrm{CD} 44$, a non-kinase transmembrane glycoprotein, which is considered to play a key role in tumorigenesis and metastasis, could directly bind to GGCT protein and lead to inhibition of its degradation.

Y-Glutamylcyclotransferase (GGCT, 188 amino acids, $21 \mathrm{kDa}$ ), an enzyme participated in glutathione metabolism, has already been detected to be up-regulated in various types of malignancies, including colorectal cancer[28], prostate cancer[29], ovarian cancer[12], and breast cancer[30]. However, few studies on GGCT have been reported in thyroid cancer research. Intriguingly, a previous study based on bioinformatics showed a seven-gene panel including GGCT exhibited significantly superior accuracy in predicting RFS and the status of immunocyte infiltration in PTC[31]. Nevertheless, it remained unknown if GGCT up-regulation is simply a concomitant product of tumor progression, or is chosen to be upregulated during cancer initiation and development, and thereby serves as an oncogenic accelerator. Our data show that the up-regulation of GGCT is not only connected with unfavorable prognosis and 
clinicopathological characteristics, but significantly affects the biological behavior of PTC and promotes its malignant conversion. As we shown in loss-of-function assays, GGCT knockdown reduced the proliferation, migration and invasion capacities and blocked EMT process of PTC cells in vitro. Further in vivo experiments strengthened that GGCT deficiency drastically inhibited tumor growth and experimental metastases. These findings highlight an indispensable role of GGCT in PTC tumorigenesis and metastasis.

Despite these promising findings, the possible mechanism as well as the upstream and downstream signaling of GGCT remain ill-defined. As previously noted in a series of reports, besides the classic oncogenes like BRAF or RET, dysregulation of miRs is implicated in the tumorigenicity and progression of PTC[21]. Among them, miR-205-5p has been proposed to exert tumor suppressor functions via repression of the downstream target gene expression, such as YAP1[32], VEGFA[22] and CCNB2[33]. In this study, we revealed GGCT is a novel target gene of miR-205-5p through the dual-luciferase reporter and RNA-RNA pull down assays. More importantly, treatment with miR-205-5p by lentiviral strategy led to a decrease expression of GGCT and reverted the malignant phenotypes mediated by GGCT. Notably, despite the expression of GGCT decreased after miR-205-5p introduction, the magnitude of that was much less pronounced than the direct interference by sh-lentivirus, inferring the existence of additional regulatory mechanisms such as competitive endogenous RNA (ceRNA) molecules between miR-205-5p and GGCT. This may also explain why overexpression of miR-205-5p alone does not effectively prevent the formation of lung metastases in experimental metastatic models as direct GGCT knockdown does. Therefore, more studies were urgent to elucidate the regulating networks between miR-205-5p and GGCT.

To further understanding the downstream mechanisms underlying GGCT-mediated pro-proliferative and pro-metastatic phenotypes, we focused then on an EMT-specific marker-CD44, whose expression was altered concomitant with GGCT. CD44 is a cell adhesion glycoprotein that plays a role in cancer progression and metastasis, and has been widely utilized as a cancer stem cell (CSC) marker in thyroid cancer[34] and various other types of malignancies[35-37]. In this study, we employed exogenous and endogenous co-IP assays and found that GGCT could bind to CD44. Further CHX chase assay demonstrated that GGCT plays a role in stabilizing CD44 to prevent its degradation. Clinically, the protein levels of GGCT were positively related to CD44 expression, demonstrating a vital GGCT-CD44 signaling axis in tumorigenesis and metastasis in PTC. Yet, exactly how GGCT binds to CD44 and affects its protein stability remains unclear. We can currently only speculate that it may be attributed to the catalytic glutaminyl cyclization function of GGCT[9]. GGCT was functionally similar to Glutaminyl cyclase (QC, Glutaminyl-peptide cyclotransferase (QPCT)) and its iso-enzyme iso-QC (QPCTL), which belong to an enzyme family that catalyze the formation of pyroglutamate ( $\mathrm{pE}-$-) at protein's N-terminus by converting glutamate/glutamine into $\mathrm{pE}-$ peptides[38]. The $\mathrm{pE}$-residue confers stability against $\mathrm{N}$-terminal degradation by proteases[39, 40]. Interestingly, a previous study by Astrid et al. found that the N-terminus of CCL2, a major chemokine involved in recruiting myeloid derived mesenchymal cells (MDSCs) to the tumor microenvironment, can be modified to a pE-residue by both QPCT and iso-QC in thyroid cancer[41]. The $\mathrm{pE}$-modification confers the resistance to proteolytic degradation of CCL2, subsequently affects tumor tumorigenesis by modulating cancer cells per se and their microenvironment niche[41]. By 
analyzing the protein sequence of CD44, a conserved glutamine at the N-terminus of CD44 (amino acid position: 21) just following the signal peptide was found, as analogous to other pE-modified molecules, suggesting the possibility of the $\mathrm{pE}$-modification of $\mathrm{CD} 44$. Further research with the aid of $\mathrm{pE}$-modifiedspecific antibodies or mass spectrometry should be conducted to confirm our conjecture.

\section{Conclusions}

In summary, we reveal a novel signaling axis, miR-205-5p/GGCT/CD44, that involves in the carcinogenesis and progression of PTC. This is the first attempt to uncover the biological function of GGCT and the mechanisms behind this protein in PTC. Usage of miR-205-mimics or GGCT inhibitors as potential therapeutics for PTC may hold great promise for clinical applications.

\section{Abbreviations}

PTC: Papillary thyroid cancer; GGCT: $\gamma$-glutamylcyclotransferase; RNAi: RNA interference; ROS: reactive oxygen species; 3'-UTR: 3 untranslated regions; qRT-PCR: quantitative real-time polymerase chain reaction; ELISA: enzyme-linked immunosorbent assays; shRNA: short hairpin RNA; CCK8: cell counting kit8; Co-IP: co-immunoprecipitation; SPF: specified-pathogen-free; fLuc: firefly-luciferase; HE: hematoxylineosin; IHC: immunohistochemistry; DAB: diaminobenzidine; EMT: epithelial-mesenchymal transition; CHX: cycloheximide; ceRNA: endogenous RNA; CSC: cancer stem cell; QPCT: Glutaminyl-peptide cyclotransferase; QPCTL: iso-glutaminyl cyclase; MDSC: myeloid derived mesenchymal cell.

\section{Declarations}

\section{Ethics approval and consent to participate}

Procedures that involved the use of human samples was approved by the Ethics Committee of Tongji Hospital, Tongji Medical College, Huazhong University of Science and Technology, and followed the ethical guidelines of the Declaration of Helsinki. The animal experiments conducted are strictly in line with the Animal Ethics Committee of Wuhan University of Science and Technology.

\section{Consent for publication}

Not applicable.

\section{Availability of data and material}

All data generated or analyzed during the study are available within the manuscript or be shared on request to the corresponding authors.

\section{Competing interests}

The authors declare that there is no conflict of interest. 


\section{Fundings}

YD is supported by National Natural Science Foundation of China (No.81802676). XRL is supported by Wuhan Youth Cadre Project (2017zqnlxr01 and 2017zqnlxr02), Clinical Research Physician Program of Tongji Medical College, HUST (5001540018) and Natural Science Foundation of Hubei Province of China (H2015038). XHL is supported by Educational Commission of Hubei (WJ2019M124), Hubei Province Health and Family Planning Scientific Research Project (WJ2021Q051), Frontier project of applied basic research in Wuhan (2020020601012250).

\section{Author contributions}

YD and XHL contributed to the conception of the study. $\mathrm{HL}$ and $\mathrm{HZ}$ performed the experiment. JW and GW contributed significantly to data analysis. SL, TX, MD, XY, YW and XC contributed to data collection and assistance in the performing of the experiment. $\mathrm{HL}, \mathrm{HZ}$ and $\mathrm{XHL}$ wrote the manuscript. YD, XRL and $\mathrm{XHL}$ contributed to the supervision of research process and provided research funding supports.

\section{Acknowledgements}

Not applicable.

\section{References}

1. Laetitia G, Sven S, Fabrice J. Combinatorial Therapies in Thyroid Cancer: An Overview of Preclinical and Clinical Progresses. Cells 2020, 9(4).

2. Wiltshire JJ, Drake TM, Uttley L, Balasubramanian SP. Systematic Review of Trends in the Incidence Rates of Thyroid Cancer. Thyroid: official journal of the American Thyroid Association. 2016;26(11):1541-52.

3. Cabanillas ME, McFadden DG, Durante C. Thyroid cancer. Lancet. 2016;388(10061):2783-95.

4. Capdevila J, Galofré JC, Grande E, Zafón Llopis C, Ramón YCAT, Navarro González E, JiménezFonseca P, Santamaría Sandi J, Gómez Sáez JM, Riesco Eizaguirre G: Consensus on the management of advanced radioactive iodine-refractory differentiated thyroid cancer on behalf of the Spanish Society of Endocrinology Thyroid Cancer Working Group (GTSEEN) and Spanish Rare Cancer Working Group (GETHI). Clinical \& translational oncology: official publication of the Federation of Spanish Oncology Societies and of the National Cancer Institute of Mexico 2017, 19(3):279-287.

5. Integrated genomic characterization of papillary thyroid carcinoma. Cell 2014, 159(3):676-690.

6. Kelly LM, Barila G, Liu P, Evdokimova VN, Trivedi S, Panebianco F, Gandhi M, Carty SE, Hodak SP, Luo $\mathrm{J}$, et al. Identification of the transforming STRN-ALK fusion as a potential therapeutic target in the aggressive forms of thyroid cancer. Proc Natl Acad Sci USA. 2014;111(11):4233-8.

7. Liu R, Xing M. TERT promoter mutations in thyroid cancer. Endocrine-related Cancer. 2016;23(3):R143-55. 
8. Martínez-Aguilar J, Clifton-Bligh R, Molloy MP. Proteomics of thyroid tumours provides new insights into their molecular composition and changes associated with malignancy. Scientific reports. 2016;6:23660.

9. Oakley AJ, Yamada T, Liu D, Coggan M, Clark AG, Board PG. The identification and structural characterization of C7orf24 as gamma-glutamyl cyclotransferase. An essential enzyme in the gamma-glutamyl cycle. J Biol Chem. 2008;283(32):22031-42.

10. Kageyama S, Iwaki H, Inoue H, Isono T, Yuasa T, Nogawa M, Maekawa T, Ueda M, Kajita Y, Ogawa O, et al. A novel tumor-related protein, C7orf24, identified by proteome differential display of bladder urothelial carcinoma. Proteomics Clinical applications. 2007;1(2):192-9.

11. Taniguchi K, Matsumura K, li H, Kageyama S, Ashihara E, Chano T, Kawauchi A, Yoshiki T, Nakata S. Depletion of gamma-glutamylcyclotransferase in cancer cells induces autophagy followed by cellular senescence. American journal of cancer research. 2018;8(4):650-61.

12. Li Y, Wu T, Wang Y, Yang L, Hu C, Chen L, Wu S. Y-Glutamyl cyclotransferase contributes to tumor progression in high grade serous ovarian cancer by regulating epithelial-mesenchymal transition via activating PI3K/AKT/mTOR pathway. Gynecol Oncol. 2018;149(1):163-72.

13. Jiang Z, Zhang C, Gan L, Jia Y, Xiong Y, Chen Y, Wang Z, Wang L, Luo H, Li J, et al. iTRAQ-Based Quantitative Proteomics Approach Identifies Novel Diagnostic Biomarkers That Were Essential for Glutamine Metabolism and Redox Homeostasis for Gastric Cancer. Proteomics Clinical applications. 2019;13(4):e1800038.

14. Kageyama S, li H, Taniguchi K, Kubota S, Yoshida T, Isono T, Chano T, Yoshiya T, Ito K, Yoshiki T, et al: Mechanisms of Tumor Growth Inhibition by Depletion of $Y$-Glutamylcyclotransferase (GGCT): A Novel Molecular Target for Anticancer Therapy. International journal of molecular sciences 2018, 19(7).

15. Taniguchi K, Kageyama S, Moyama C, Ando S, li H, Ashihara E, Horinaka M, Sakai T, Kubota S, Kawauchi A, et al: $\mathrm{Y}$-Glutamylcyclotransferase, a novel regulator of HIF-1a expression, triggers aerobic glycolysis. Cancer gene therapy 2021.

16. He Z, Wang S, Shao Y, Zhang J, Wu X, Chen Y, Hu J, Zhang F, Liu XS: Ras Downstream Effector GGCT Alleviates Oncogenic Stress. iScience 2019, 19:256-266.

17. Vosgha H, Ariana A, Smith RA, Lam AK. miR-205 targets angiogenesis and EMT concurrently in anaplastic thyroid carcinoma. Endocrine-related Cancer. 2018;25(3):323-37.

18. He L, Hannon GJ. MicroRNAs: small RNAs with a big role in gene regulation. Nature reviews Genetics. 2004;5(7):522-31.

19. Hu X, Wang Y, Liang H, Fan Q, Zhu R, Cui J, Zhang W, Zen K, Zhang CY, Hou D, et al. miR-23a/b promote tumor growth and suppress apoptosis by targeting PDCD4 in gastric cancer. Cell death disease. 2017;8(10):e3059.

20. Zhao J, Li L, Yang T. MiR-216a-3p suppresses the proliferation and invasion of cervical cancer through downregulation of ACTL6A-mediated YAP signaling. Journal of cellular physiology. 2020;235(12):9718-28. 
21. Mastronikolis N, Tsiambas E, Roukas D, Fotiades P, Chrysovergis A, Papanikolaou V, Kyrodimos E, Mastronikoli S, Niotis A, Ragos V. Micro-RNAs signatures in papillary thyroid carcinoma. Journal of BUON: official journal of the Balkan Union of Oncology. 2020;25(5):2144-6.

22. Salajegheh A, Vosgha H, Md Rahman A, Amin M, Smith RA, Lam AK. Modulatory role of miR-205 in angiogenesis and progression of thyroid cancer. J Mol Endocrinol. 2015;55(3):183-96.

23. Yang J, Huang Y, Dong B, Dai Y: Long noncoding RNA DLEU2 drives the malignant behaviors of thyroid cancer through mediating the miR-205-5p/TNFAIP8 axis. Endocrine connections 2021, 10(4):471-483.

24. Zhou J, Cao L, Chen Z. Differentiation of benign thyroid nodules from malignant thyroid nodules through miR-205-5p and thyroid-stimulating hormone receptor mRNA. Hormones (Athens Greece). 2021;20(3):571-80.

25. Chen C, Zhao S, Karnad A, Freeman JW. The biology and role of CD44 in cancer progression: therapeutic implications. J Hematol Oncol. 2018;11(1):64.

26. Pastushenko I, Blanpain C. EMT Transition States during Tumor Progression and Metastasis. Trends in cell biology. 2019;29(3):212-26.

27. Takahashi RU, Prieto-Vila M, Kohama I, Ochiya T. Development of miRNA-based therapeutic approaches for cancer patients. Cancer Sci. 2019;110(4):1140-7.

28. Huang Q, Zhou Y, Li Y, Liao Z. GGCT promotes colorectal cancer migration and invasion via epithelial-mesenchymal transition. Oncology letters. 2020;20(2):1063-70.

29. Takagi H, li H, Kageyama S, Hanada E, Taniguchi K, Yoshiya T, Chano T, Kawauchi A, Nakata S. Blockade of $\mathrm{y}$-Glutamylcyclotransferase Enhances Docetaxel Growth Inhibition of Prostate Cancer Cells. Anticancer research. 2019;39(9):4811-6.

30. Matsumura K, Nakata S, Taniguchi K, li H, Ashihara E, Kageyama S, Kawauchi A, Yoshiki T. Depletion of $Y$-glutamylcyclotransferase inhibits breast cancer cell growth via cellular senescence induction mediated by CDK inhibitor upregulation. BMC Cancer. 2016;16(1):748.

31. Zhang L, Wang Y, Li X, Wang Y, Wu K, Wu J, Liu Y. Identification of a Recurrence Signature and Validation of Cell Infiltration Level of Thyroid Cancer Microenvironment. Front Endocrinol. 2020;11:467.

32. Li D, Wang Q, Li N, Zhang S. miR-205 targets YAP1 and inhibits proliferation and invasion in thyroid cancer cells. Mol Med Rep. 2018;18(2):1674-81.

33. Wang X, Zhang H, Jiao K, Zhao C, Liu H, Meng Q, Wang Z, Feng C, Li Y. Effect of miR-205 on proliferation and migration of thyroid cancer cells by targeting CCNB2 and the mechanism. Oncology letters. 2020;19(3):2568-74.

34. De Falco V, Tamburrino A, Ventre S, Castellone MD, Malek M, Manié SN, Santoro M. CD44 proteolysis increases CREB phosphorylation and sustains proliferation of thyroid cancer cells. Cancer research. 2012;72(6):1449-58.

35. Gomez KE, Wu F, Keysar SB, Morton JJ, Miller B, Chimed TS, Le PN, Nieto C, Chowdhury FN, Tyagi A, et al. Cancer Cell CD44 Mediates Macrophage/Monocyte-Driven Regulation of Head and Neck 
Cancer Stem Cells. Cancer research. 2020;80(19):4185-98.

36. Martincuks A, Li PC, Zhao Q, Zhang C, Li YJ, Yu H, Rodriguez-Rodriguez L. CD44 in Ovarian Cancer Progression and Therapy Resistance-A Critical Role for STAT3. Frontiers in oncology. 2020;10:589601.

37. Ding R, Li G, Yao Y, Zhang L, Zhang X, Li J, Shen T, Gao Y, Wu T, Kong S, et al: Transgelin-2 interacts with CD44 to regulate Notch1 signaling pathway and participates in colorectal cancer proliferation and migration. Journal of physiology and biochemistry 2021.

38. Stephan A, Wermann M, von Bohlen A, Koch B, Cynis H, Demuth HU, Schilling S. Mammalian glutaminyl cyclases and their isoenzymes have identical enzymatic characteristics. FEBS J. 2009;276(22):6522-36.

39. Logtenberg MEW, Jansen JHM, Raaben M, Toebes M, Franke K, Brandsma AM, Matlung HL, Fauster A, Gomez-Eerland R, Bakker NAM, et al. Glutaminyl cyclase is an enzymatic modifier of the CD47SIRPa axis and a target for cancer immunotherapy. Nature medicine. 2019;25(4):612-9.

40. Wu Z, Weng L, Zhang T, Tian H, Fang L, Teng H, Zhang W, Gao J, Hao Y, Li Y, et al. Identification of Glutaminyl Cyclase isoenzyme isoQC as a regulator of SIRPa-CD47 axis. Cell research. 2019;29(6):502-5.

41. Kehlen A, Haegele M, Menge K, Gans K, Immel UD, Hoang-Vu C, Klonisch T, Demuth HU. Role of glutaminyl cyclases in thyroid carcinomas. Endocrine-related Cancer. 2013;20(1):79-90.

\section{Figures}


A

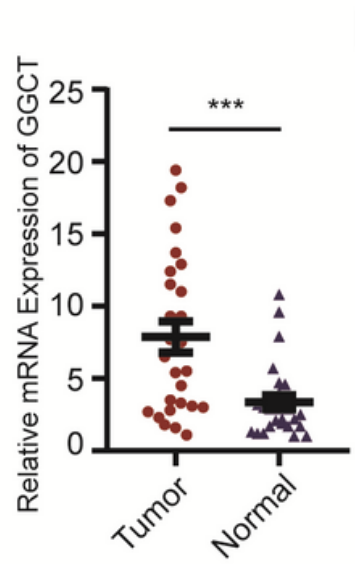

B

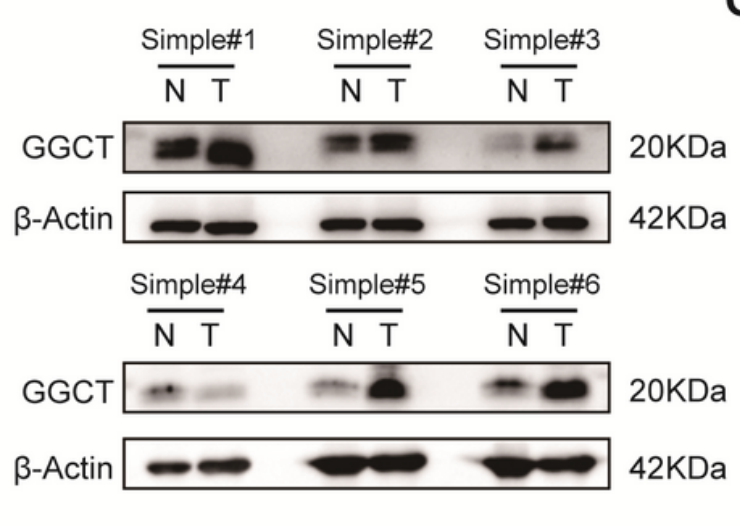

D
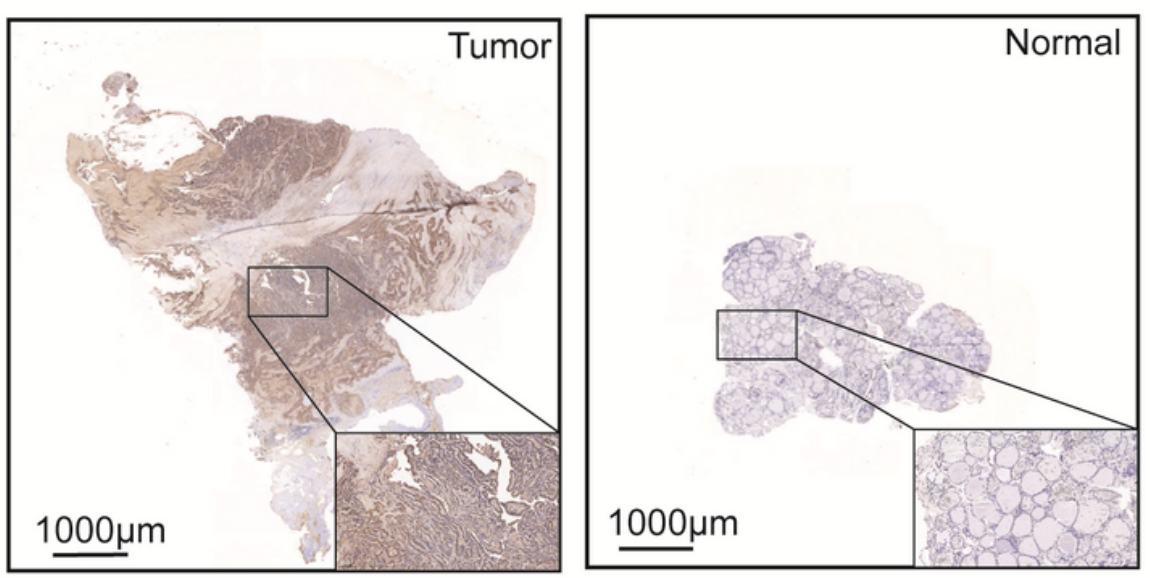

F

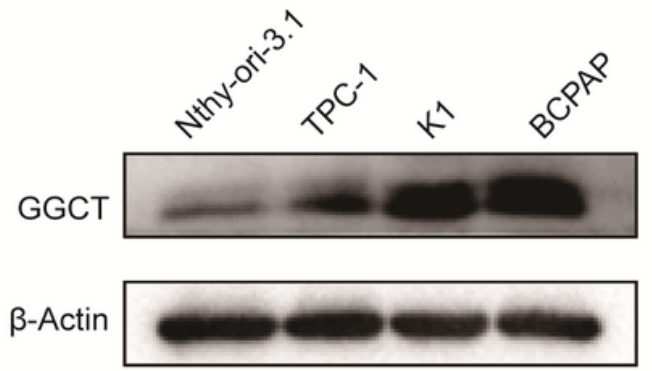

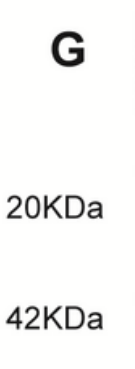

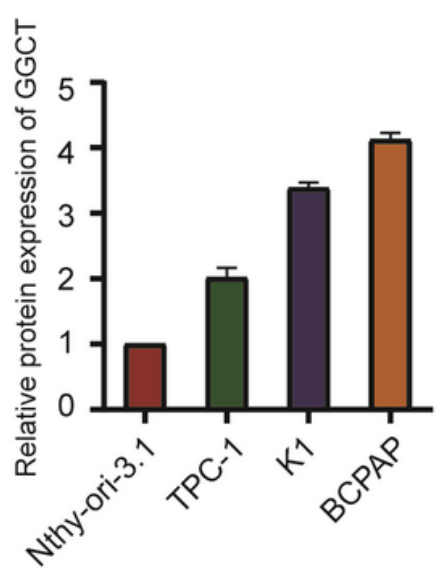

C

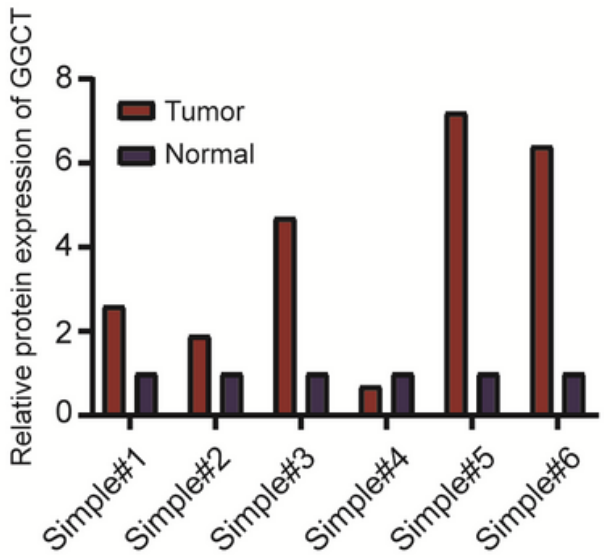

$\mathbf{E}$

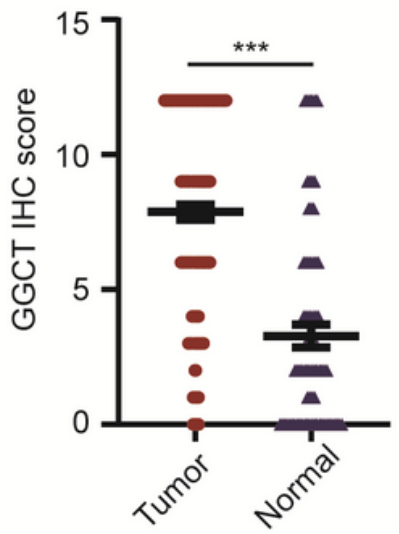

H

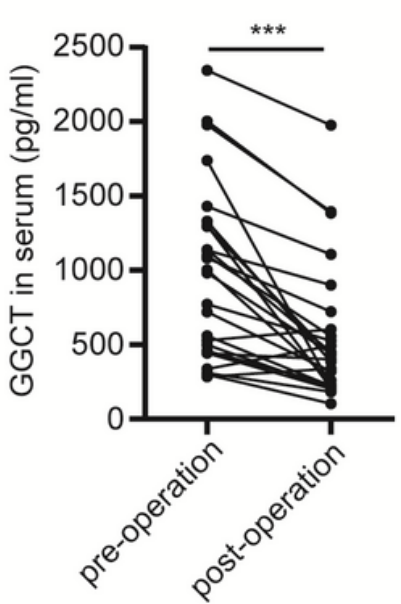

\section{Figure 1}

GGCT is overexpressed in PTC. (A) Scatter dot plots show the mRNA expression of GGCT in PTC and normal thyroid tissues $(n=27)$. (B) Western Blot (WB) experiments show the protein expression of GGCT in six paired normal and tumor samples. (C) The quantifications of WB signal in each patient were normalized to $\beta$-Actin and presented as histograms. (D) Representative images of immunohistochemistry (IHC) of GGCT in the normal thyroid tissues (right) and PTC tissues (left) (magnification, $\times 100$, scar bar $=1000 \mu \mathrm{m})$. Solid black boxes indicate higher magnifications $(\times 400)$ of the areas. (E) IHC scores of GGCT were calculated in PTC group $(n=178)$ and normal thyroid group $(n=82)$. GGCT protein levels in 
PTC and normal thyroid cell lines were assessed by WB (F) and quantified (G). (H) The level of secreted GGCT in patient's serum before and $24 \mathrm{~h}$ after surgical intervention was determined by ELISA assays $(n=26)$. The line connecting the two scatters denote the change in GGCT secretion of the same patient before and after surgery. ${ }^{* *}: \mathrm{p}<0.001$.

A
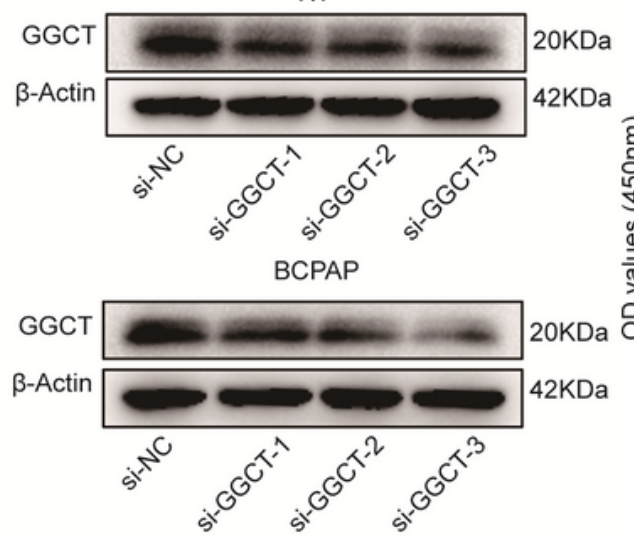

D

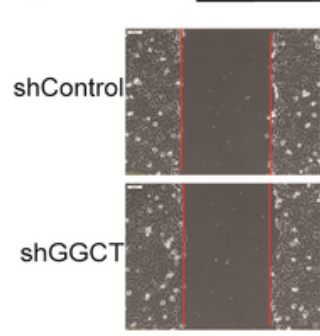

Oh

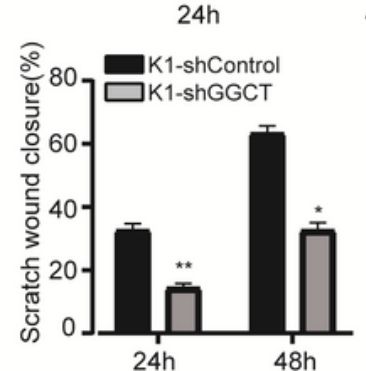

F

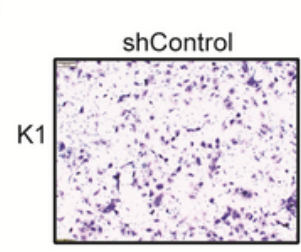

G
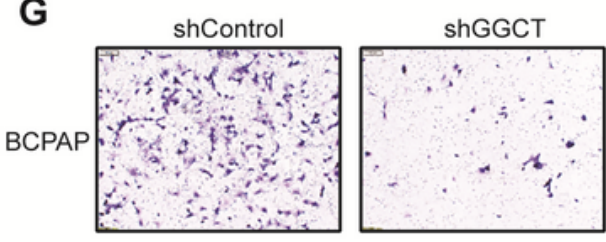

B 20KDa $42 \mathrm{KDa}$

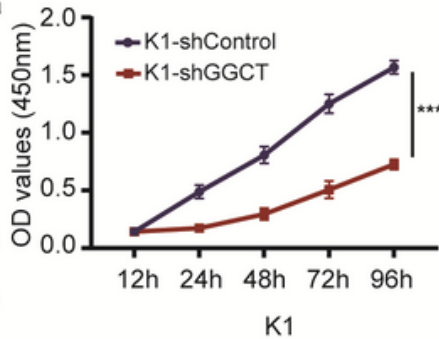

K1

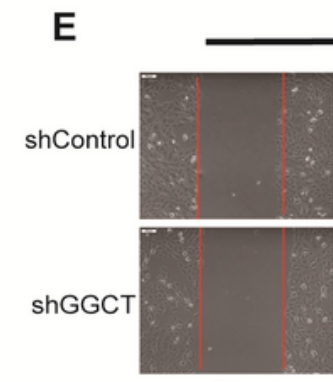

Oh

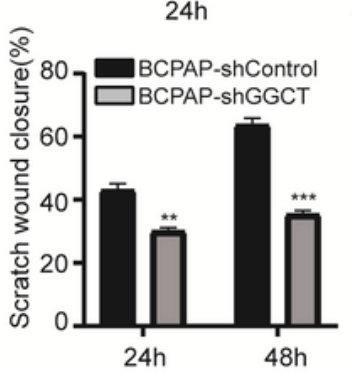

$8 \mathrm{~h}$
BCPAP

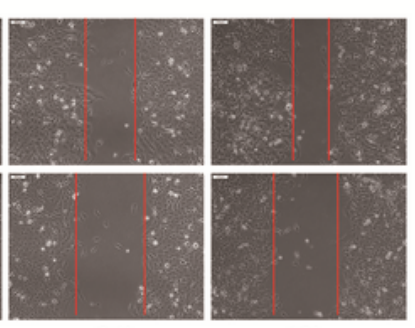

$48 \mathrm{~h}$
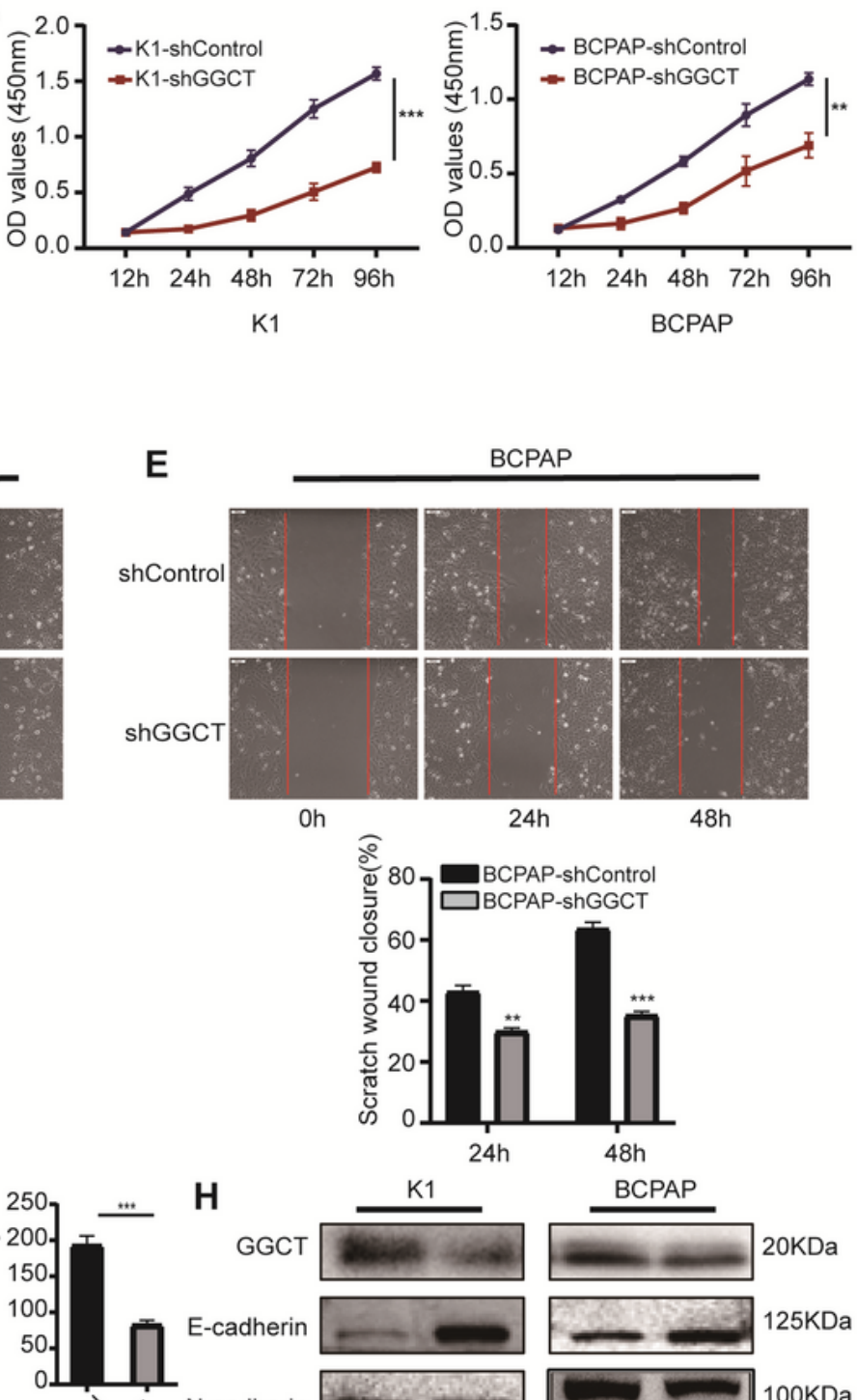

H

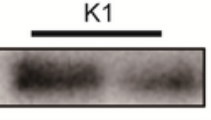

BCPAP

GGCT

$\mathrm{E}$-cadherin
$\mathrm{N}$-cadherin
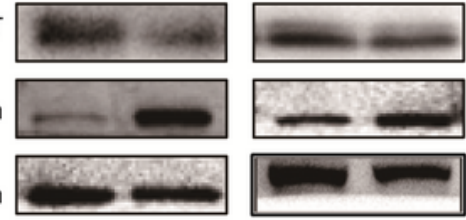

$20 \mathrm{KDa}$
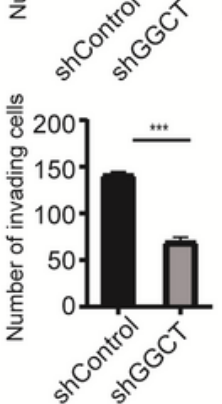

CD44
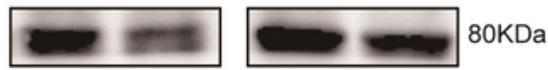

MMP2
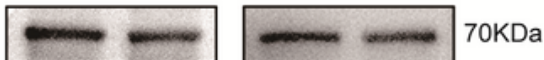

MMP9
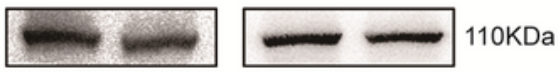

$\beta$-Actin
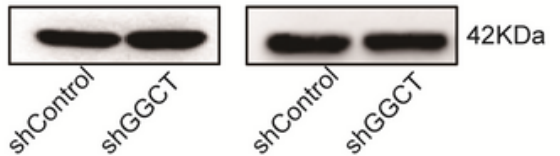

Figure 2 
Downregulation of GGCT decreases PTC cell proliferation and aggressiveness in vitro. (A) WB analysis validation for GGCT knockdown in K1 and BCPAP cells. (B-C) Cell proliferation determined by CCK-8 in K1 and BCPAP cells infected with sh-GGCT or sh-Control as indicated. (D-E) Wound healing assay comparing the migration ability between sh-GGCT and sh-Control PTC cells. (F-G) Transwell assay of K1 and BCPAP cells infected with sh-GGCT or sh-Control. $(\mathrm{H})$ The abundance of EMT-related markers Ecadherin, N-cadherin, CD44, MMP-2 and MMP-9 were measured by WB in the K1 and BCPAP cells following GGCT knockdown. *: $p<0.05, * *$ : $p<0.01, * \star *$ : $p<0.001$.

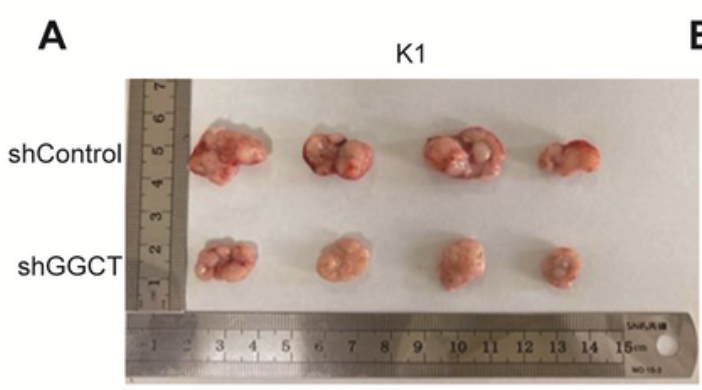

B

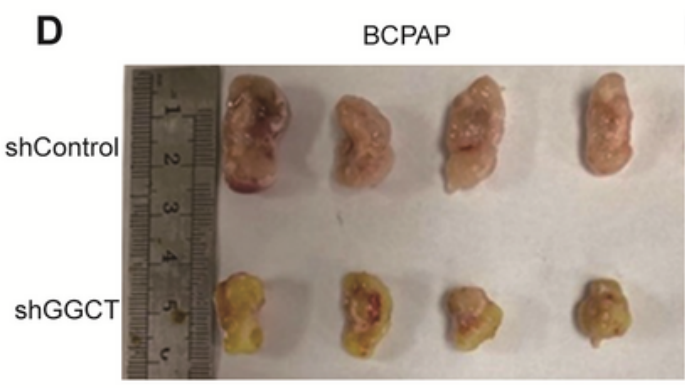

E

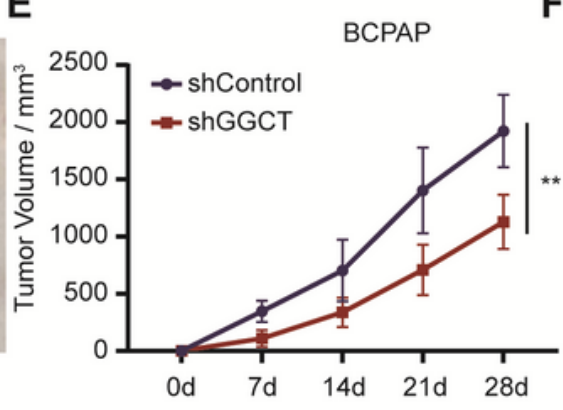

C

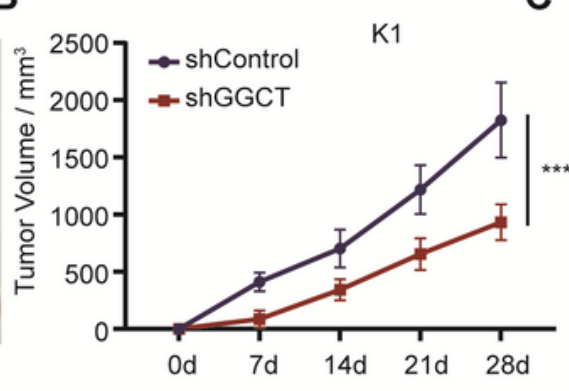

F
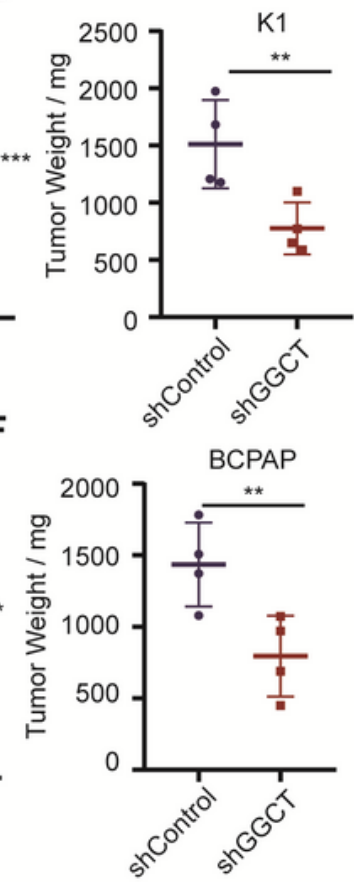
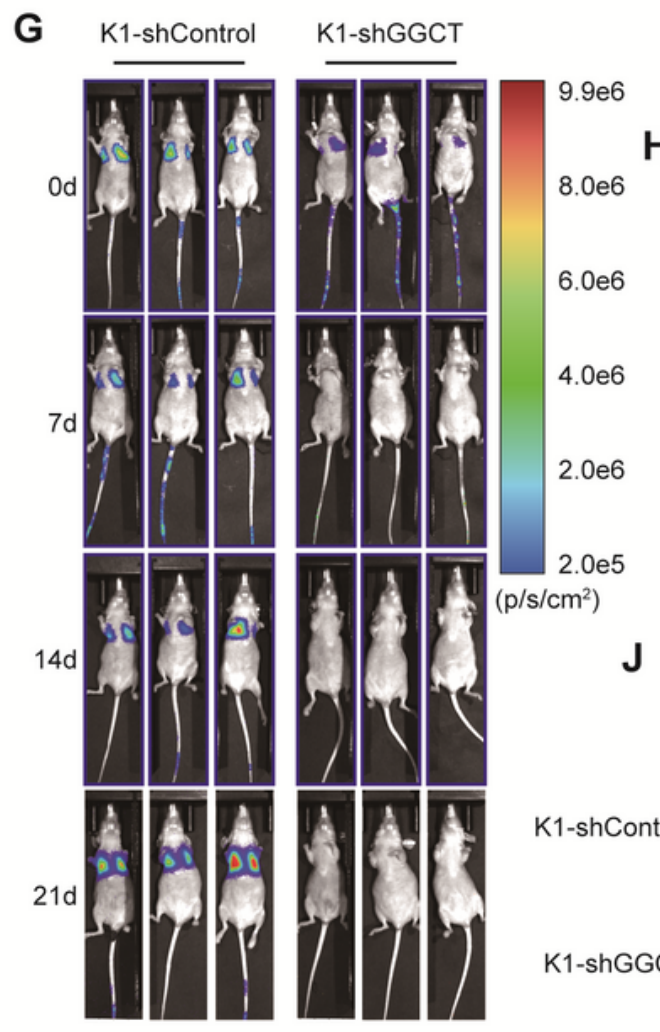

I

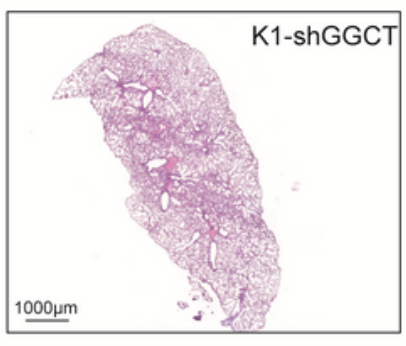

-

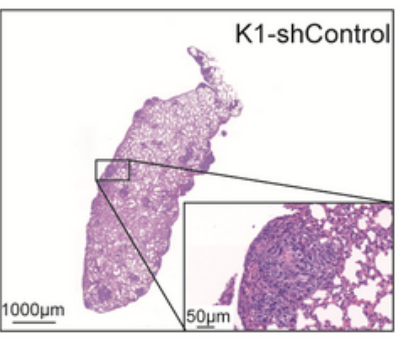

K1-shControl

K1-shGGCT

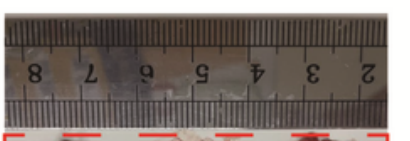



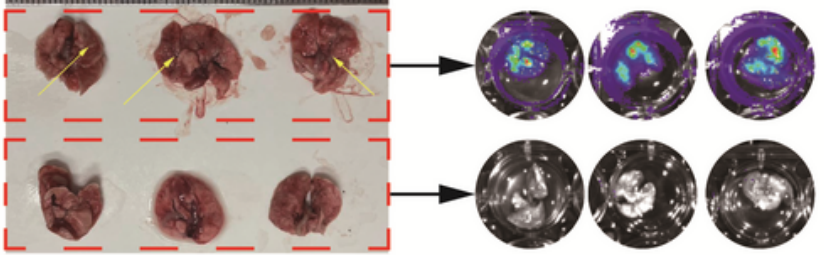




\section{Figure 3}

GGCT knockdown delays tumor growth and suppresses lung metastasis of PTC cells in vivo. (A and D) Representative images of K1 and BCPAP subcutaneous xenografts from nude mice in sh-GGCT and shControl groups. (B and E) Tumor volumes were measured every 7 days post implantation. ( $C$ and $F$ ) Comparison of dissected tumor weights in each group 28 days post tumor implantation. (G) Metastasis bioluminescence images of K1-shControl and K1-shGGCT every 7 days post tumor cell tail vein injection. $(\mathrm{H})$ The line chart denotes luciferase bioluminescence emitted from the lungs in each group over time (021 days). (I) HE staining for the lungs of K1-shControl and K1-shGGCT mice. The figure boxed in the lower right corner shows the metastatic foci with higher magnifications. (J) Gross anatomy (left) and ex vivo bioluminescence (right) images from K1-shControl and K1-shGGCT mice at day 21 after tumor cells challenge. $* *: p<0.01, * \star *: p<0.001$. 
A

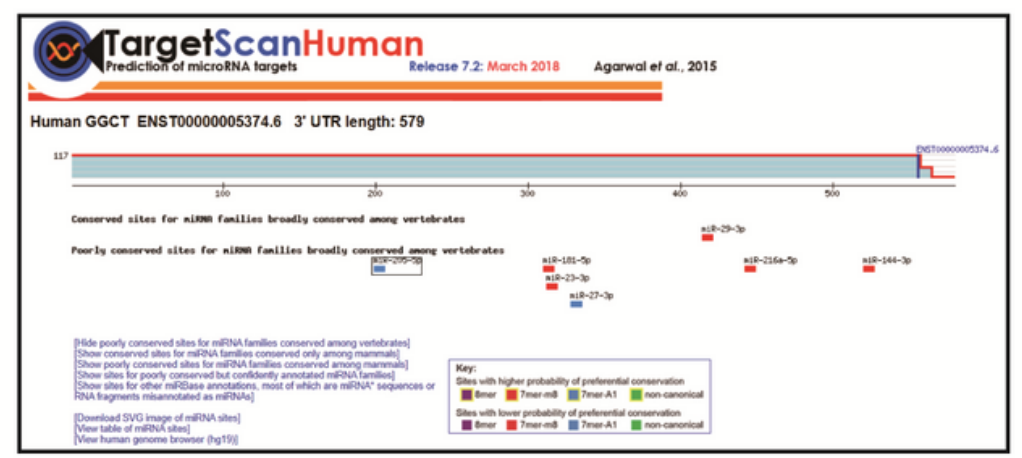

C

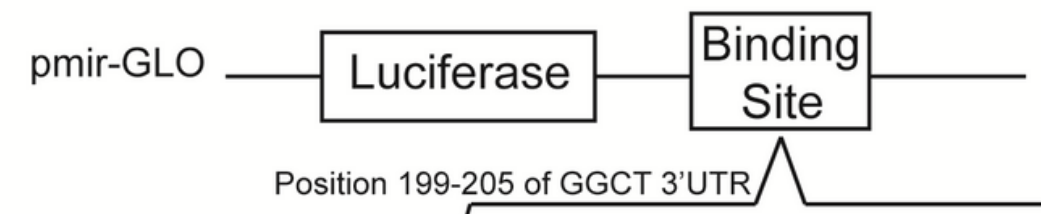

WT-pmirGLO-GGCT ...CAGCAGTGCTCTGAAGGA...

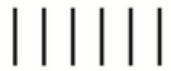

miR-205-5p

...GAGGCCACCUUACUUCCU...

MUT-pmirGLO-GGCT ...CAGCAGTGCTCCAGTACA...

E

Bio-miR-205-5p-WT ...GAGGCCACCUUACUUCCU...

GGCT 3'UTR

...CAGCAGTGCTCTGAAGGA...

Bio-miR-205-5p-WUT...GAGGCCACCUUCAGGAAU...
B

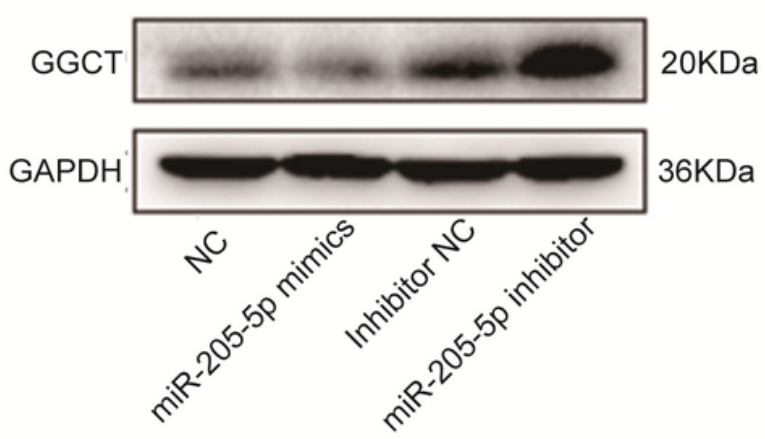

D

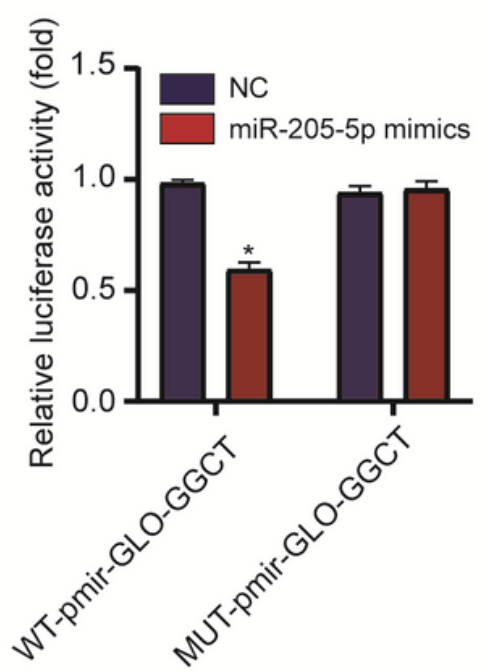

$\mathbf{F}$

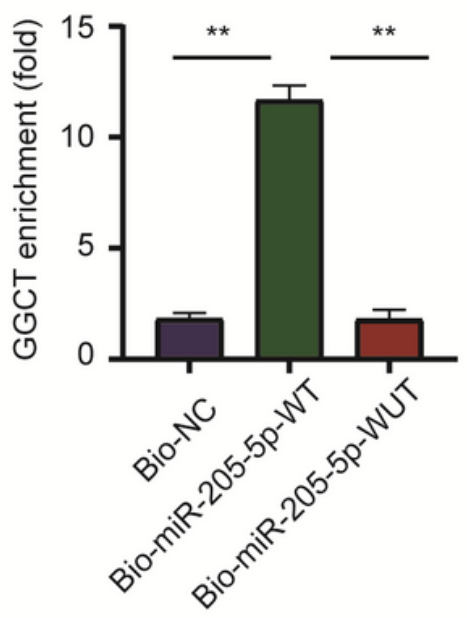

\section{Figure 4}

MiR-205-5p binds to 3'-UTR of GGCT and regulates the expression of GGCT. (A) Position of the miR-205$5 p$ target site in 3'-UTR of human GGCT mRNA predicted by TargetScan (http://www.targetscan.org/). (B) Western blot analysis of the effect of miR-205-5p mimics or inhibitors on GGCT protein levels in K1 cells. (C) Schematic representation of the binding sites of miR-205-5p and the expression vectors construction of the wild type and mutant GGCT- 3'UTR pmir-GLO plasmids used in luciferase reporter assays. (D) Luciferase reporter vectors WT-pmirGLO-GGCT (intact) or WUT-pmirGLO-GGCT (mutant) were transfected 
in $\mathrm{K} 1$ cells. The luciferase activity was measured and the values were normalized to Renilla luciferase activity. (E) Schematic of biotin-labeled RNA-RNA pull down assays. (F) Expression of GGCT mRNA in biotin-labeled miRNA/mRNA complex was demonstrated by qRT-PCR. *: $p<0.05$, **: $p<0.01$.

A

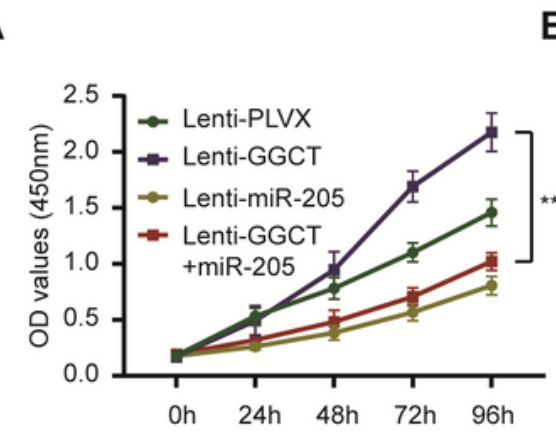

B Lenti-PLVX +

C

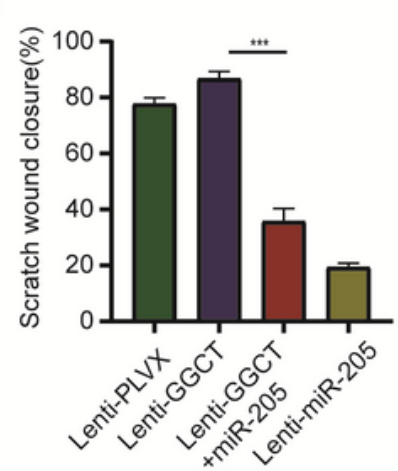

\section{D}

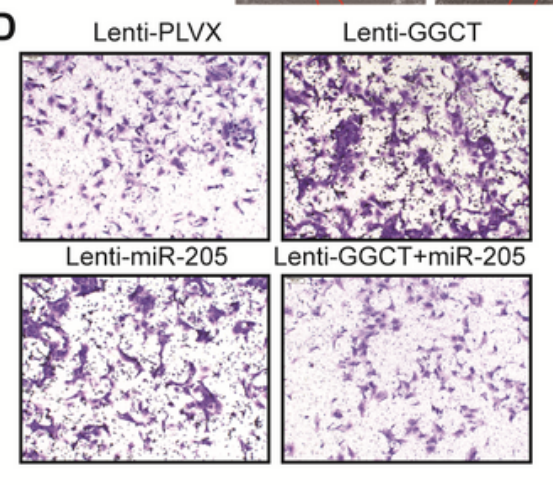

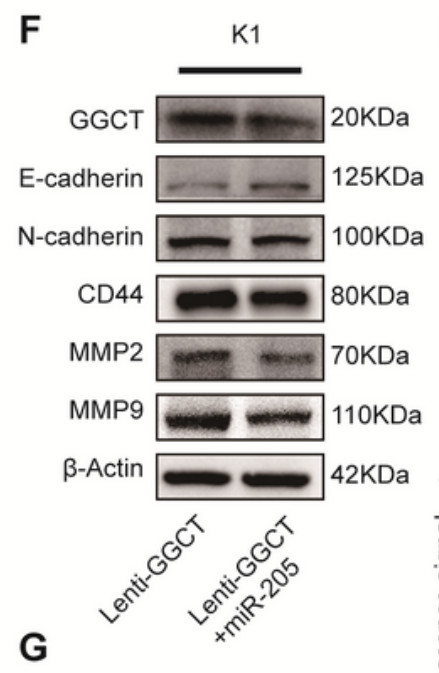

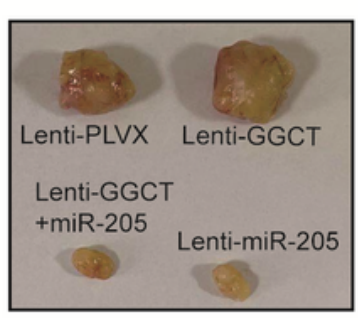

H
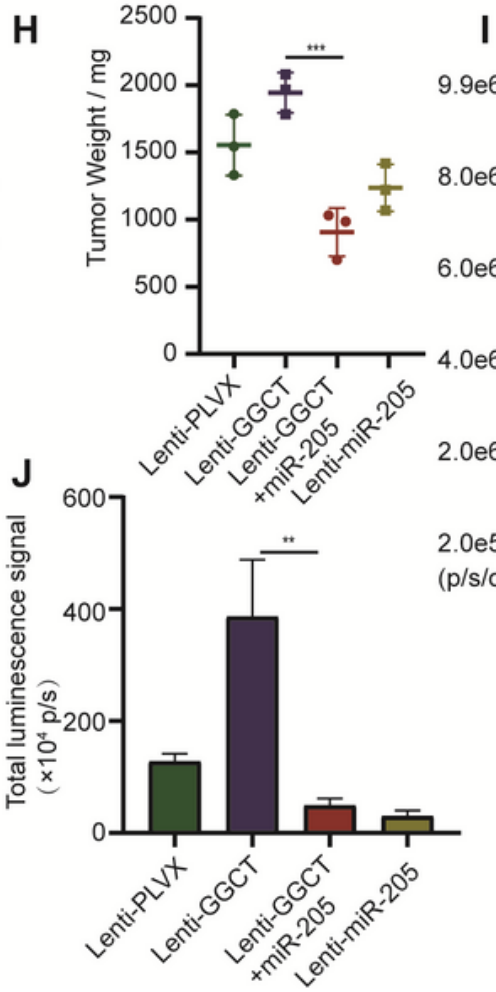

E
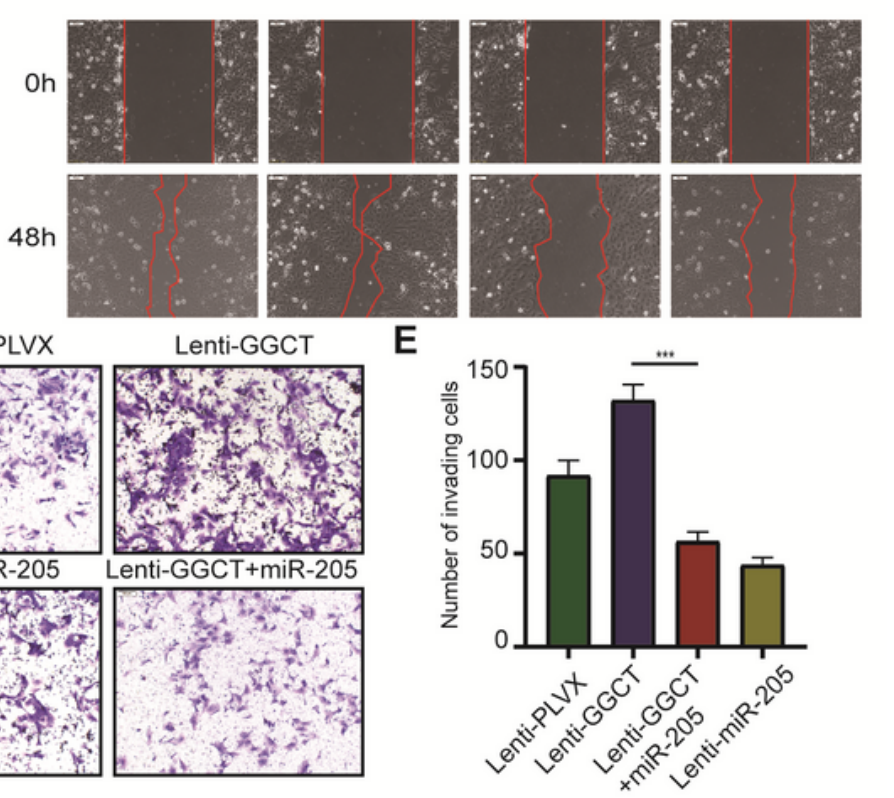

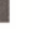

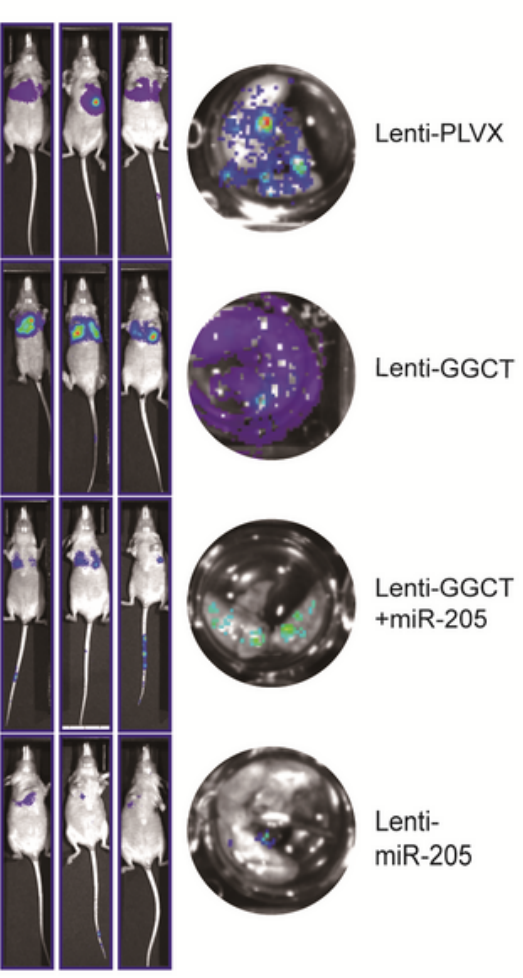

Figure 5

Induction of miR-205-5p attenuates GGCT-mediated PTC cell proliferation, migration, invasion, EMT process, tumorigenic ability, and distant metastatic potential to the lung. (A) Cell viability was examined 
by CCK8 after overexpression of miR-205-5p in K1-Control or K1-GGCT cell lines. Cell migration (B-C) and invasion (D-E) potentials were determined by wound healing and transwell assays. ( $F$ ) Expression of epithelial marker (E-cadherin) and mesenchymal markers (N-cadherin, CD44, MMP-2 and MMP-9) was analyzed by Western blotting after miR-205-5p overexpression in K1-GGCT cells. (G) Tumorigenic ability was assessed after miR-205-5p overexpression in K1-GGCT cells. (H) Dissected tumor weights in each group were depicted as dot plots. (I) Tumor lung metastasis was assayed after miR-205-5p overexpression in K1-GGCT cells. $(\mathrm{J})$ The histogram denotes luciferase bioluminescence emitted from the lungs in each group 21 days post tumor injection. $* *$ : $p<0.01$, $* \star *: p<0.001$. 
A

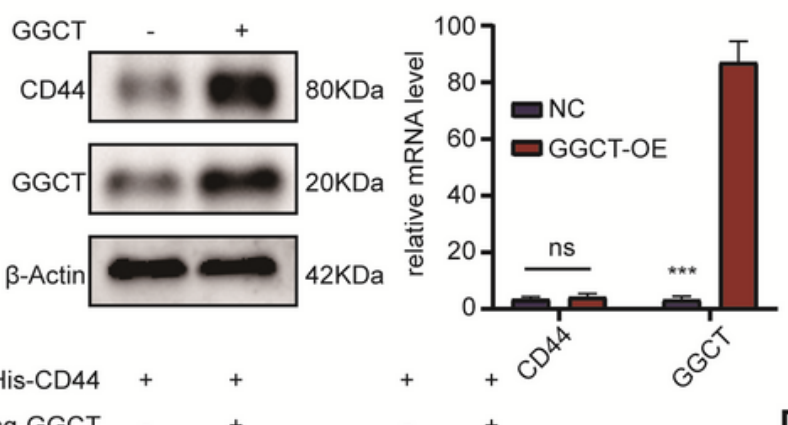

Flag-GGCT

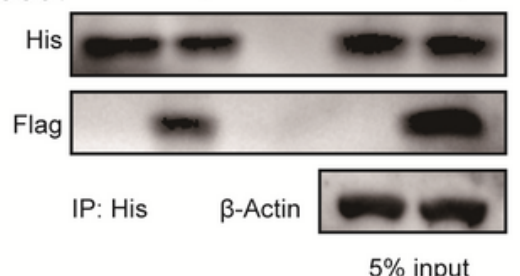

B
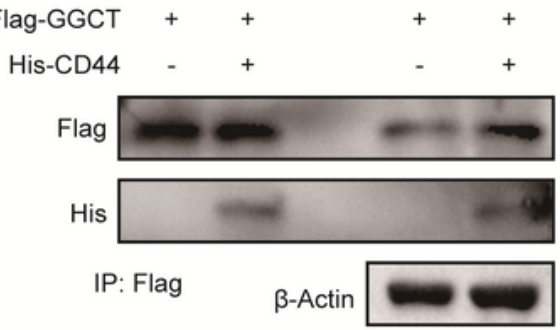

$5 \%$ input
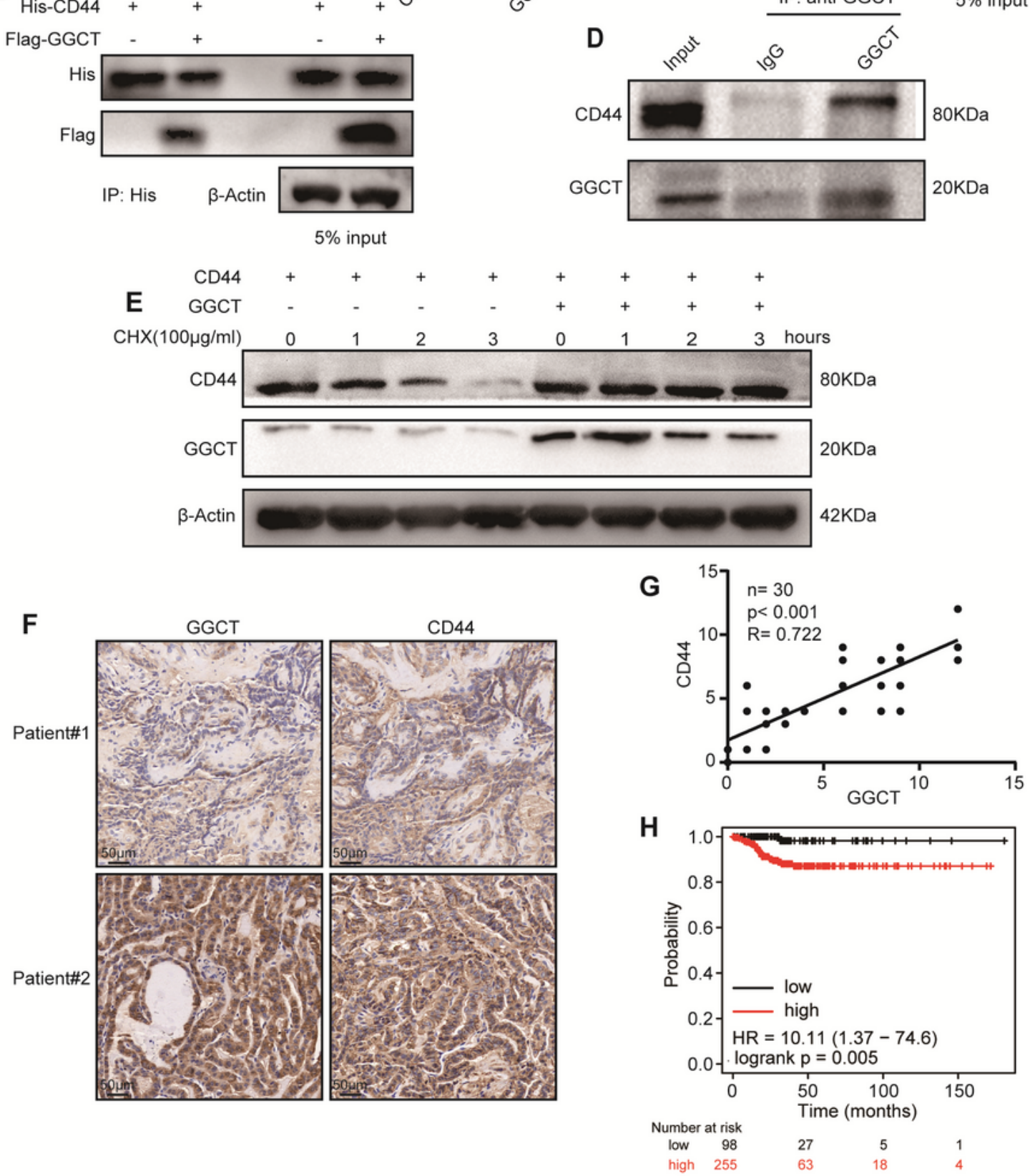

\section{Figure 6}

GGCT interacts with and stabilizes CD44. (A) K1 cells were transfected with GGCT or control vectors. The western blot showed the protein level of GGCT and CD44 (left) and the RT-qPCR was conducted to measure the mRNA level of GGCT and CD44 (right). (B and C) Flag-GGCT or vector plasmids were coexpressed with His-CD44 or vector plasmids in HEK-293T cells. Cell lysates were immunoprecipitated with anti-Flag or anti-His antibodies, followed by immunoblotting. (D) The endogenous interaction of GGCT 
and CD44 in K1 cells was detected by Co-IP and western blot assays. (E) CD44 was co-expressed with vector or GGCT in $\mathrm{K} 1$ cells. After $24 \mathrm{~h}$, cells were challenged with $\mathrm{CHX}(100 \mu \mathrm{g} / \mathrm{ml})$ for $0-3 \mathrm{~h}$, followed by western blot assays. (F) Representative images of GGCT and CD44 IHC staining in tumor sections from the same patient. (G) The figure shows the correlation between GGCT and CD44 IHC scores, with the Pearson correlation coefficients $(R)$, p value as well as simple numbers $(n)$ in the upper corner. $(H)$ The DFS of CD44 expression in PTC. The analysis was conducted using the K-M Plotter online tool (http://kmplot.com/analysis/). ***: $p<0.001$.

\section{Supplementary Files}

This is a list of supplementary files associated with this preprint. Click to download.

- TableS1.docx

- figures1.tif

- figures2.tif

- FigureS3.tif 